\title{
BARYON DYNAMICS, DARK MATTER SUBSTRUCTURE, AND GALAXIES
}

\author{
DAVID H. WeINBERG \\ Astronomy Department, Ohio State University, Columbus, OH 43210; dhw@astronomy.ohio-state.edu \\ Stephane Colombi \\ Institut d'Astrophysique de Paris, 75014 Paris, France; colombi@iap.fr \\ ROMEel DAvé \\ Steward Observatory, University of Arizona, Tucson, AZ 85721; rad@as.arizona.edu \\ AND \\ NeAL KatZ \\ Astronomy Department, University of Massachusetts, Amherst, MA 01003; nsk@kaka.astro.umass.edu \\ Received 2006 April 14; accepted 2007 September 13
}

\begin{abstract}
By comparing a collisionless cosmological $N$-body simulation (DM) to a smoothed particle hydrodynamics simulation (SPH) with the same initial conditions, we investigate the correspondence between dark matter subhalos produced by collisionless dynamics and galaxies produced by dissipative gas dynamics in a dark matter background. When galaxies in the SPH simulation fall into larger groups and become satellites, they retain local dark matter concentrations (SPH subhalos) whose mass is typically 5 times the galaxy baryonic mass (compared to the simulation's universal ratio $\Omega_{\mathrm{dm}} / \Omega_{b} \approx 7.5$ ). The more massive subhalos of the SPH simulation generally have corresponding subhalos of similar mass and spatial position in the DM simulation; at lower masses, there is still fairly good correspondence, but some DM subhalos are in different spatial positions and some have suffered tidal stripping or disruption. The halo occupation statistics of DM subhalos - the mean number of subhalos, pairs, and triples as a function of host halo mass - are very similar to those of SPH subhalos and SPH galaxies. The gravity of the dissipative baryon component amplifies the density contrast of subhalos in the SPH simulation, making them more resistant to tidal disruption. Relative to SPH galaxies and SPH subhalos, the DM subhalo population is depleted in the densest regions of the most massive halos. The good agreement of halo occupation statistics between the DM subhalo and SPH galaxy populations leads to good agreement of their two-point correlation functions and higher order moments on large scales. The depletion of DM subhalos in dense regions depresses their clustering at $R<1 h^{-1} \mathrm{Mpc}$. In these simulations, the "conversation" between dark matter and baryons is mostly one-way, with dark matter dynamics telling galaxies where to form and how to cluster, but the "back talk" of the baryons influences small-scale clustering by enhancing the survival of substructure in the densest environments.
\end{abstract}

Subject headings: galaxies: formation — large-scale structure of universe

Online material: color figures

\section{INTRODUCTION}

The idea that galaxies form by dissipation of the baryonic component within a collisionless dark matter halo has a long history (White \& Rees 1978). The excellent agreement of the inflationary cold dark matter (CDM) model with a wide range of cosmological observations (e.g., Spergel et al. 2003; Seljak et al. 2005) puts this hypothesis on a firm theoretical footing. In the first stages of galaxy formation, dark matter does the talking: gravitational collapse produces dark matter potential wells that capture baryons, which radiate their energy and form dense objects at the halo centers. However, the subsequent details of the baryon-dark matter "conversation" are not so clear. Early $N$-body simulations showed that mergers of dark matter halos were followed by fairly rapid erasure of substructure, suggesting that dissipation in the baryonic component was crucial to the formation of groups and clusters with many distinct members, and that it was the gravity of the condensed baryons that allowed them to retain the central regions of their individual dark matter halos after falling into larger virialized systems. However, much higher resolution simulations in the late 1990s and thereafter showed that virialized halos retain a great deal of substructure (Ghigna et al. 1998; Klypin et al. 1999;
Moore et al. 1999; Springel et al. 2001), and that the erasure of substructure in earlier simulations was largely an artifact of inadequate mass and/or force resolution. This result raises the possibility that baryon self-gravity is unimportant in producing groups and clusters, and that cooling and star formation merely produce "beacons" that mark the centers of dark matter structures that would be present even if baryons had no gravitational influence at all.

In this paper we investigate the dynamical interaction between baryons and dark matter using two numerical simulations of the same cosmological volume, one that incorporates both dark matter and a baryonic component modeled with smoothed particle hydrodynamics (SPH), and the other that starts from the same initial conditions but follows only the dark matter. We identify substructure in the dark matter distribution using a method that computes SPH-like density estimates within halos, then groups particles above saddle points in the density field. We are interested in the degree to which the presence of baryons alters the properties of substructure in the dark matter distribution and in the degree to which substructure in the purely gravitational simulation traces the galaxy population that forms in the hydrodynamic simulation. The latter issue is of practical as well of physical 
interest, since if the agreement is good one might be able to use $\mathrm{N}$-body simulations in place of hydrodynamic simulations for galaxy clustering predictions. $N$-body simulations with the resolution needed to follow substructure are computationally expensive, but they are less expensive than full hydrodynamic simulations, thus allowing larger simulation volumes or wider searches of parameter space. We therefore pay particular attention to the halo occupation statistics of substructures versus galaxies, since these in turn allow one to predict many different clustering statistics (Berlind \& Weinberg 2002).

There are several indirect indications that substructure in $N$-body simulations can provide good tracers of the galaxy population. First, Colín et al. (1999) and Kravtsov et al. (2004) show that the correlation functions of substructures in high-resolution simulations agree quite well with observations, which in turn agree well with results from full hydrodynamic simulations (Cen \& Ostriker 2000; Pearce et al. 2001; Yoshikawa et al. 2001; Weinberg et al. 2004). Conroy et al. (2006) show that the agreement with observations extends to a wide range of redshifts and galaxy space densities. Second, Berlind et al. (2003) show that there is remarkably good agreement between the halo occupation distribution found in our SPH simulations and those predicted by semianalytic galaxy formation models of Cole et al. (2000). Since the treatment of cooling and star formation is quite different in the two methods, this agreement suggests that the halo occupation distribution is determined in large part by dark matter dynamics and halo merger histories, although even if true this does not guarantee that postmerger substructure will retain the information about the galaxy population. Third, Kravtsov et al. (2004) find that the halo occupation distribution of substructure in their high-resolution $N$-body simulations is similar to that found in our SPH simulations by Berlind et al. (2003) and Zheng et al. (2005). While all of these results provide useful insight into the relative importance of dark matter dynamics and baryon dissipation in determining the spatial distribution of galaxies, this paper is the first, to our knowledge, to carry out the direct test of comparing galaxy populations in a hydrodynamic simulation of a cosmological volume to dark matter substructure in an $N$-body simulation with the same initial conditions. Nagai \& Kravtsov (2005) have recently carried out a complementary experiment in simulations of galaxy clusters, and Macciò et al. (2006) have carried out an analogous experiment on the scale of a single, galaxy-mass halo $\left(M \approx 10^{12} M_{\odot}\right)$, focusing on lower mass substructures than those considered here.

\section{SIMULATIONS}

We analyze two simulations with the same initial conditions, one run with dark matter only and one that incorporates a dissipative gas component and star formation. We hereafter refer to these as the DM and SPH simulations, respectively. The SPH simulation uses a parallel implementation (Davé et al. 1997) of TreeSPH (Hernquist \& Katz 1989; Katz et al. 1996) to follow the evolution of $128^{3}$ dark matter particles and $128^{3}$ gas particles in a $22.222 h^{-1}$ Mpc comoving box, from $z=49$ to $z=0$. We adopt a $\Lambda$ CDM cosmological model (inflationary cold dark matter with a cosmological constant) with parameters $\Omega_{m}=0.4, \Omega_{\Lambda}=0.6$, $\Omega_{b}=0.02 h^{-2}, h \equiv H_{0} / 100 \mathrm{~km} \mathrm{~s}^{-1} \mathrm{Mpc}^{-1}=0.65, n=0.95$, and $\sigma_{8}=0.8$. Our choices of $\sigma_{8}, H_{0}, n$, and $\Omega_{b}$ are reasonably close to the recent estimates from cosmic microwave background anisotropies and large-scale structure data (e.g., Spergel et al. 2003; Tegmark et al. 2004; Sánchez et al. 2006), while our value of $\Omega_{m}$ is somewhat high. The dark matter particle mass is $7.9 \times$ $10^{8} M_{\odot}$, and the SPH particle mass is $1.05 \times 10^{8} M_{\odot}$. The gravitational force softening is a $5 h^{-1}$ comoving kpc cubic spline, roughly equivalent to a Plummer force softening of $3.5 \mathrm{~h}^{-1}$ comoving kpc. The DM simulation uses the same simulation code and the same initial positions and velocities of dark matter particles. It has the same numerical parameters, except that the dark matter particle mass is increased by a factor of $\Omega_{m} /\left(\Omega_{m}-\Omega_{b}\right)$ to $8.9 \times 10^{8} M_{\odot}$.

Although the volume is much smaller, the mass resolution of the DM simulation is similar to that of the simulations used by Colín et al. (1999) and Kravtsov et al. (2004) to study the largescale clustering of subhalos (somewhat higher than their $\Lambda \mathrm{CDM}_{60}$ run with $256^{3}$ particles in a $60 h^{-1} \mathrm{Mpc}$ box and somewhat lower than their $\Lambda \mathrm{CDM}_{80}$ run with $512^{3}$ particles in an $80 h^{-1} \mathrm{Mpc}$ box), and it is slightly higher than that of the recent "Millenium Run" simulation (Springel et al. 2005; $2160^{3}$ particles in a $500 h^{-1} \mathrm{Mpc}$ box). It is not as high as the mass resolution in recent simulations focused on the substructure distribution in clusters (e.g., Diemand et al. 2004; Gao et al. 2004; Nagai \& Kravtsov 2005). By chance, our simulation forms one halo that is unusually large for a $22.222 \mathrm{~h}^{-1} \mathrm{Mpc}$ box given our cosmological parameters, with a mass of $4 \times 10^{14} M_{\odot}$, allowing us to investigate substructure survival and baryonic influence in a Virgo-mass galaxy cluster (see Fig. 3 below). This halo contains about $4.7 \times 10^{5}$ dark matter particles within its virial region. The next most massive halos have masses of $\sim 3 \times 10^{13} M_{\odot}$.

Details of the treatment of radiative cooling, star formation, and galaxy identification in the SPH simulation can be found in Katz et al. (1996). In brief, gas can dissipate energy via Compton cooling and radiative cooling, computed assuming primordial composition and the photoionizing background field of Haardt \& Madau (1996). Star formation occurs in regions that are Jeans unstable, above a threshold density $\left(n_{\mathrm{H}}>0.1 \mathrm{~cm}^{-3}\right)$, and below a threshold temperature $(T \leq 30,000 \mathrm{~K})$. We add the thermal energy from supernova feedback but it has relatively little impact, because it is usually deposited in a dense medium with a short cooling time. We identify galaxies using the Spline Kernel Interpolative DENMAX (SKID) ${ }^{1}$ algorithm (Gelb \& Bertschinger 1994; Katz et al. 1996), which identifies gravitationally bound clumps of stars and cold $(T \leq 30,000 \mathrm{~K})$, dense $\left(\rho_{g} / \bar{\rho}_{g} \geq 1000\right)$ gas that are associated with a common density maximum. Comparisons among simulations with different resolution show that the locations and baryonic masses (stars plus cold, dense gas) of SKID galaxies are robust when the mass exceeds that of about 64 SPH particles, or $6.8 \times 10^{9} M_{\odot}$. For our lowest mass threshold sample in this paper, we take a slightly larger minimum mass of $7.1 \times 10^{9} M_{\odot}$. There are 1103 galaxies in the simulation volume above this mass, making the mean space density of this sample $0.1 \mathrm{~h}^{3} \mathrm{Mpc}^{-3}$. We also consider samples with higher minimum masses and lower mean space densities, as discussed in $\S 4$ below.

\section{IDENTIFICATION OF SUBSTRUCTURE}

Our method of identifying halos and substructures uses the publicly available code AdaptaHOP (Aubert et al. 2004), which derives from the HOP algorithm of Eisenstein \& Hut (1998). It is very similar to the method used by Springel et al. (2001) known as SUBFIND, although the implementation here is independent. The algorithm is detailed in Aubert et al. (2004), so we give only a brief summary of it here. We first calculate densities around each dark matter particle using an SPH-like kernel estimator, with a cubic spline kernel containing 32 neighbors (just as in the SPH simulation itself). During that operation, we store as well the $N_{\text {hop }}$ nearest neighbors of each particle, with $N_{\text {hop }}=16$ as advocated by

\footnotetext{
1 We use the implementation of SKID by J. Stadel and T. Quinn, which is publicly available at http://www-hpcc.astro.washington.edu/tools/skid.html.
} 


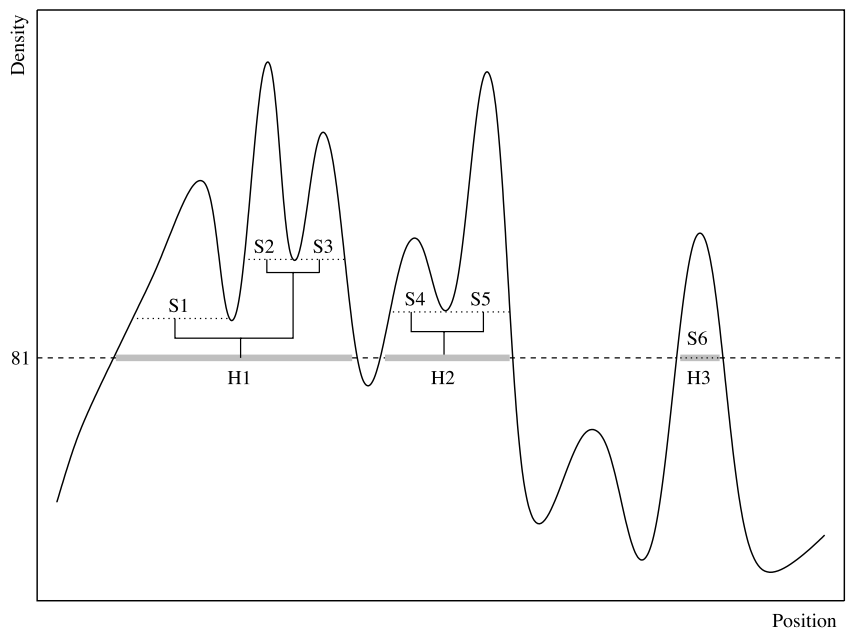

FIg. 1.-Illustration of AdaptaHOP, in one dimension. In this example, saddle points are local minima. The halos are connected structures above the density threshold. Three of them are detected, H1, H2, and H3, as defined by the thick horizontal lines. Each elementary substructure (a leaf) is associated to a local maximum. Therefore, $\mathrm{H} 1, \mathrm{H} 2$, and $\mathrm{H} 3$ contain respectively 3,2 and 1 leaves. A given substructure can be connected to other ones by saddle points. For instance S2 is connected to $\mathrm{S} 1$ and $\mathrm{S} 3$ through two saddle points. To compute the extension of each substructure, we take only the points which have density larger than $\rho_{s}$, where $\rho_{s}$ is the maximum value measured at the saddle points, as defined by the horizontal dotted lines. Note that halo H3 is its own substructure, S6. Its boundary is defined by the halo density threshold $\rho / \bar{\rho}=81$. [See the electronic edition of the Journal for a color version of this figure.]

Eisenstein \& Hut (1998). Then, we partition the ensemble of particles into "peak patches." A peak patch is a set of particles with the same local density maximum, identified by connecting each particle to its densest neighbor among its $N_{\text {hop }}$ closest to track the local density gradient. The connectivity between the peak patches is dictated by the saddle points in the density field. These points are found by identifying local maxima in the boundaries between peak patches. In fact, for each pair of peak patches connected through at least one saddle point, one needs only the saddle point with highest density. Then one is ready to construct an ensemble of trees, the halos, and the branches of the trees and their leaves, the substructures, each leaf corresponding to a unique local maximum, or peak patch.

In this representation, a halo is defined as a connected group of particles with overdensity $\rho / \bar{\rho}>81$, as advocated by Eisenstein $\&$ Hut (1998); a leaf is defined as a subset of particles in a peakpatch with SPH density larger than $\rho_{s}$, where $\rho_{s}$ is the density of the highest saddle point connecting this peak patch to a neighboring one. In order to select substructures which are statistically significant compared to Poisson noise, we impose a $4 \sigma$ level threshold,

$$
\langle\rho\rangle_{\mathrm{sub}}>\rho_{\mathrm{s}}\left(1+\frac{4}{\sqrt{N}}\right)
$$

where $N$ is the number of particles in this substructure (with SPH density above $\rho_{S}$ ) and $\langle\rho\rangle_{\text {sub }}$ is the average SPH density in this substructure. A substructure not following this constraint is absorbed by the neighboring substructure connected to it through the highest saddle point. This operation is performed recursively until condition (1) is fulfilled. Finally, note that most of halos do not have any substructure, or equivalently, only one, the halo itself. In our representation, and in what follows, such a halo is considered simultaneously as a halo and a substructure. A higher resolution simulation would presumably reveal substructures in these low-mass halos, but they would be below the mass threshold of the resolved galaxy populations that we consider below.

To understand the procedure followed in AdaptaHOP, it is instructive to look at the one-dimensional analog shown in Figure 1. The densities at the particle locations define a one-dimensional density field. Halos are connected regions above the overdensity threshold of 81, and there are three such halos in Figure 1. Saddle points in three dimensions correspond to local minima in this one-dimensional example, and the three halos in Figure 1 contain three substructures, two substructures, and no substructure, respectively. There is necessarily one and only one maximum between each pair of minima, and this maximum is identified as the location of the substructure. The mass of the substructure is the mass above the density threshold of the higher minimum, as indicated by the horizontal dotted lines in Figure 1. In most cases, this is a reasonable way of assigning mass, but it tends to underestimate the mass of a large central object with a much smaller satellite. Note that the sum of the masses of the substructures in a halo is generally smaller than the halo mass itself, unless the halo does not contain any substructure. In the present paper, we use only the trees (halos) and leaves (individual subhalos), but the substructure finder also builds the entire set of branches using saddle points as connectors.

\section{RESULTS}

\subsection{Formation of a Galaxy Group}

Figure 2 illustrates the formation history of a representative galaxy group. This group occupies the fourth most massive halo in the simulation, with a mass of $3.1 \times 10^{13} M_{\odot}$. The left and middle columns show the dark matter particle distributions in the DM and SPH simulations, respectively, with particles color-coded according to local density estimated with the 32 particle spline kernel used in the substructure identification. Specifically, the bottom panels show the dark matter particle distributions in the central $0.5 h^{-1} \mathrm{Mpc}$ of this halo at $z=0$; the full extent of the region within the $\rho / \bar{\rho}>81$ surface is about a factor of 2 larger. The top three rows show the distributions of the same particles at $z=2$, 1 , and 0.5 , respectively (a small number of particles are missed off the bottom of these plots). At $z=2$, these particles are spread over a region roughly $5 h^{-1} \mathrm{Mpc}$ across (comoving), and many of them are clumped into small halos aligned along filamentary structures. Between $z=2$ and $z=0.5$, the small halos merge into larger halos, the underlying filamentary network becomes less evident, and the whole comoving volume shrinks slightly in size.

The right-hand column of Figure 2 shows the distribution of star particles, shown as green dots, and gas particles, color coded by temperature, from the SPH simulation. Note that high density can make the stellar clumps visually inconspicuous even when they are fairly massive; we have used larger dots for star particles at $z \geq 0.5$ so that the clumps remain visible. By $z=0$, all of the gas that is not in galaxies has been heated to $T \sim 5 \times 10^{6} \mathrm{~K}$, but at earlier times much of the gas in filaments or the diffuse medium between them is cooler than $10^{5} \mathrm{~K}$. The high-redshift panels also show clumps of dense gas that has cooled to $T \sim 10^{4} \mathrm{~K}$ but has not yet formed stars. The absence of stars in these clumps is primarily a numerical resolution effect - in tests with simulations of varying resolution, we find that the SPH code underestimates star formation rates in objects with less than $\sim 200$ particles.

At $z \geq 1$, the dark matter distributions in the simulations with and without gas are nearly indistinguishable, and even at $z=$ 0.5 , the differences are small. Gas condensation and star formation occur at the centers of the larger dark matter concentrations. At $z=0$, all of the larger galaxies are associated with a visually 

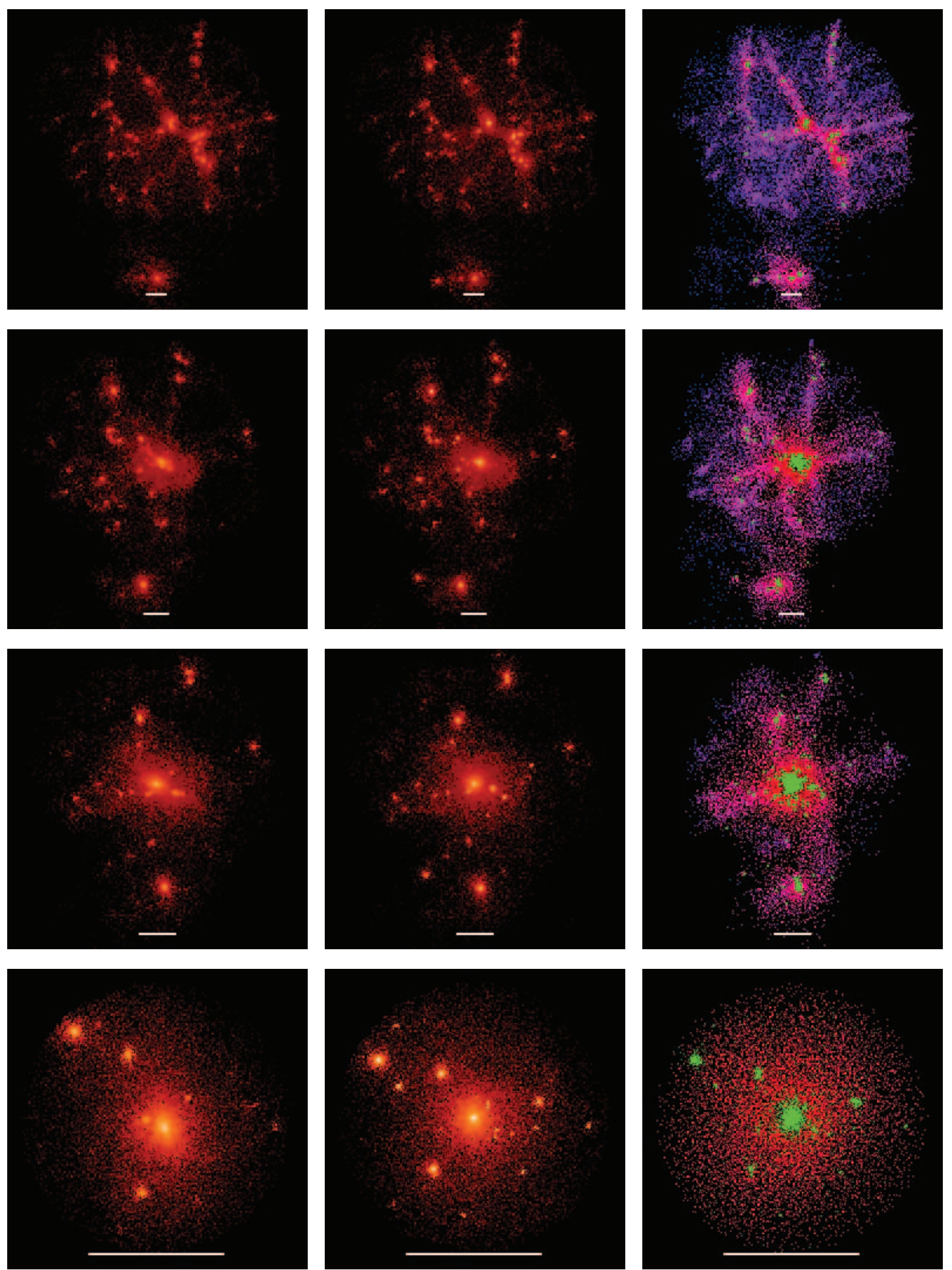

FIG. 2.-Formation of a galaxy group, in a halo of mass $3.1 \times 10^{13} M_{\odot}$. The bottom left panel shows the dark matter particles in the central $0.5 h^{-1} \mathrm{Mpc}$ of this halo at $z=0$, in the DM simulation. Panels above it show the positions of the same particles at $z=3,1$, and 0.5 (top to bottom). The panel size is different at each redshift; the white bar is always $0.5 h^{-1} \mathrm{Mpc}$ comoving. Central panels show the corresponding dark matter particle distributions in the SPH simulation. Right-hand panels show the distributions of gas and star particles at the same redshifts. Green points (plotted larger for visibility at $z>0$ ) show star particles. Gas particles are color coded by temperature on a scale running from $\sim 5 \times 10^{3} \mathrm{~K}$ (blue) to $\sim 5 \times 10^{6} \mathrm{~K}$ (red).

identifiable dark matter substructure. The largest substructures are at similar locations in the DM and SPH simulations. Smaller substructures cannot be visually matched one-to-one between the two simulations. The gravity of the dense baryon clumps increases the density of subhalos in the SPH simulation, making them more visually prominent. However, we will show below that the number and mass distribution of subhalos is actually similar in the SPH and DM simulations, and that the level of one-to-one subhalo correspondence is more than meets the eye.

\subsection{Galaxy and Subhalo Populations}

Figure 3 shows the largest halo in the simulation, with a mass of $4.0 \times 10^{14} M_{\odot}$. The left-hand panels show the dark matter particle distribution in the SPH simulation (top) and DM simulation 

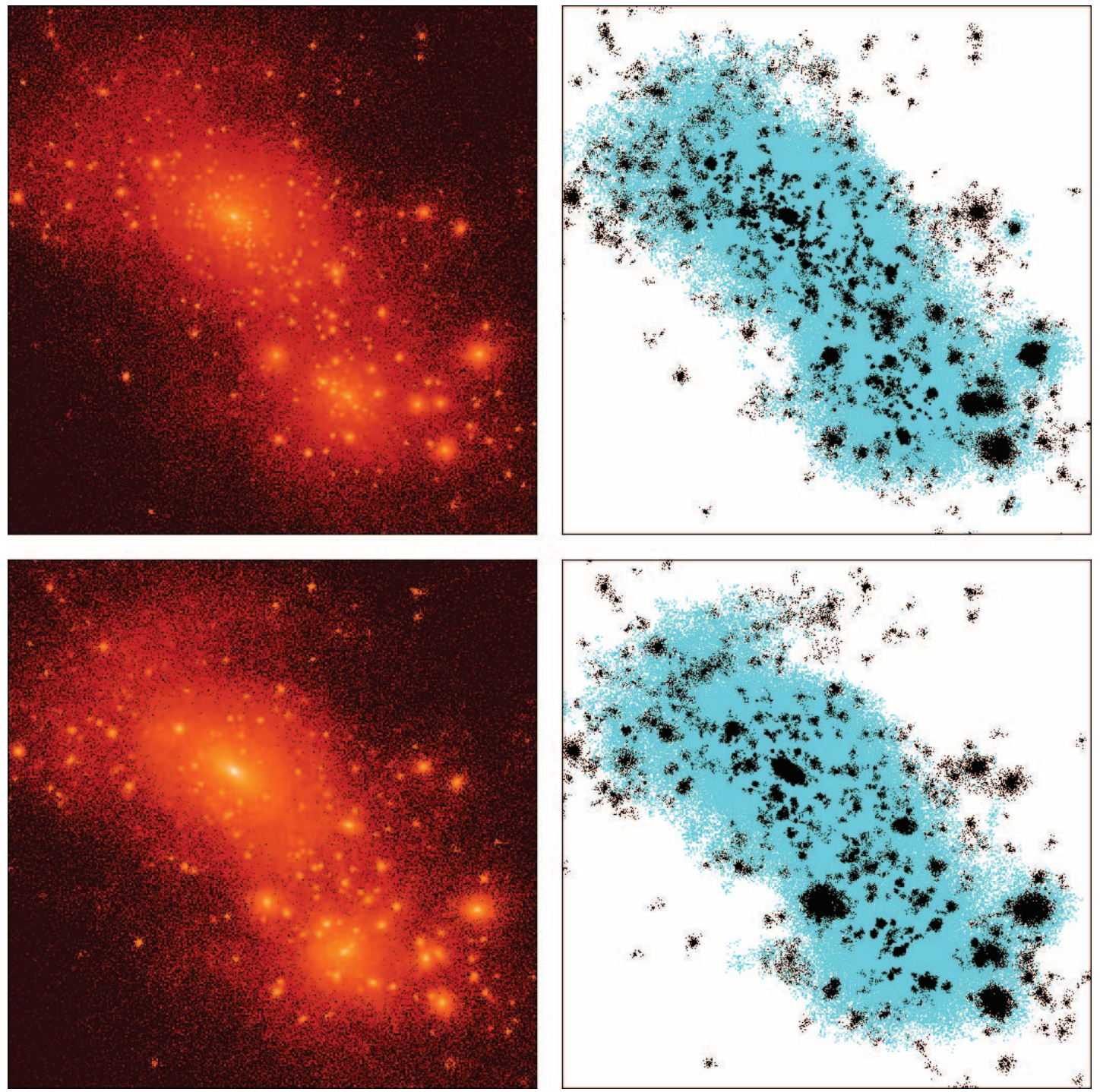

FIG. 3. - Dark matter and dark matter substructure for the largest halo in the simulation, with a mass of $4.0 \times 10^{14} M_{\odot}$ at $z=0$. Panels are $4 h^{-1}$ Mpc across. Left-hand panels show the halo dark matter particles in the SPH simulation (top) and DM simulation (bottom). Particles are coded by local density, estimated using a spline kernel enclosing 32 neighbors. In the right-hand panels, black points show particles that are members of subhalos identified by AdaptaHOP. Cyan points show particles that are not connected to one of these substructures but are above the overdensity 81 threshold.

(bottom), with particles color-coded according to their local density, again estimated with the 32 particle spline-kernel smoothing used in the substructure identification. The halo contains two major subcomponents within its $\rho / \bar{\rho}=81$ density boundary. One can see a large number of local density maxima in both the SPH and DM simulations. There is good correspondence in the positions of the larger density maxima, while the smaller density peaks are similar in number but do not correspond in position. One can also see that these local density peaks are systematically suppressed in the DM simulation near the centers of the two large clumps. A higher resolution simulation might preserve a larger degree of substructure in these innermost regions, but the SPH and DM simulations have the same mass and force resolution, so the differential effect of including the dissipative baryon component should be correct, at least qualitatively. Several groups have recently carried out detailed convergence tests for cluster substructure in dissipationless $N$-body simulations and concluded that the suppression of substructure in cluster cores is for the most part a real effect of tidal stripping and disruption rather than a numerical artifact (Diemand et al. 2004; Gao et al. 2004). It is also possible that higher gravitational force resolution would increase the retention of substructure in our SPH simulation by producing denser baryonic cores, since our spline softening of $5 h^{-1} \mathrm{kpc}$ is comparable to the expected size of angular momentum supported disks; Macciò et al. (2006) find a significant effect in this direction in their substructure study. However, our tests in previous studies show that the baryonic masses of galaxies with $M_{b} \gtrsim 64 M_{\mathrm{SPH}}$ are stable to changes in mass and force resolution (see, e.g., Keres et al. 2005), and since the baryonic core is in any event much denser than the dark subhalo we do not expect the effect in our simulations to be large.

The right-hand panels of Figure 3 illustrate the application of AdaptaHOP to this halo. Black points show particles that have been assigned to a subhalo, and cyan points show particles that are assigned to the parent halo but not to a subhalo. One can see a good correspondence between the positions of the largest identified subhalos in the two simulations, as expected from the density maps. It is hard to infer the mass of substructures from these plots because of saturation; denser subhalos often appear less massive because they are more compact. 

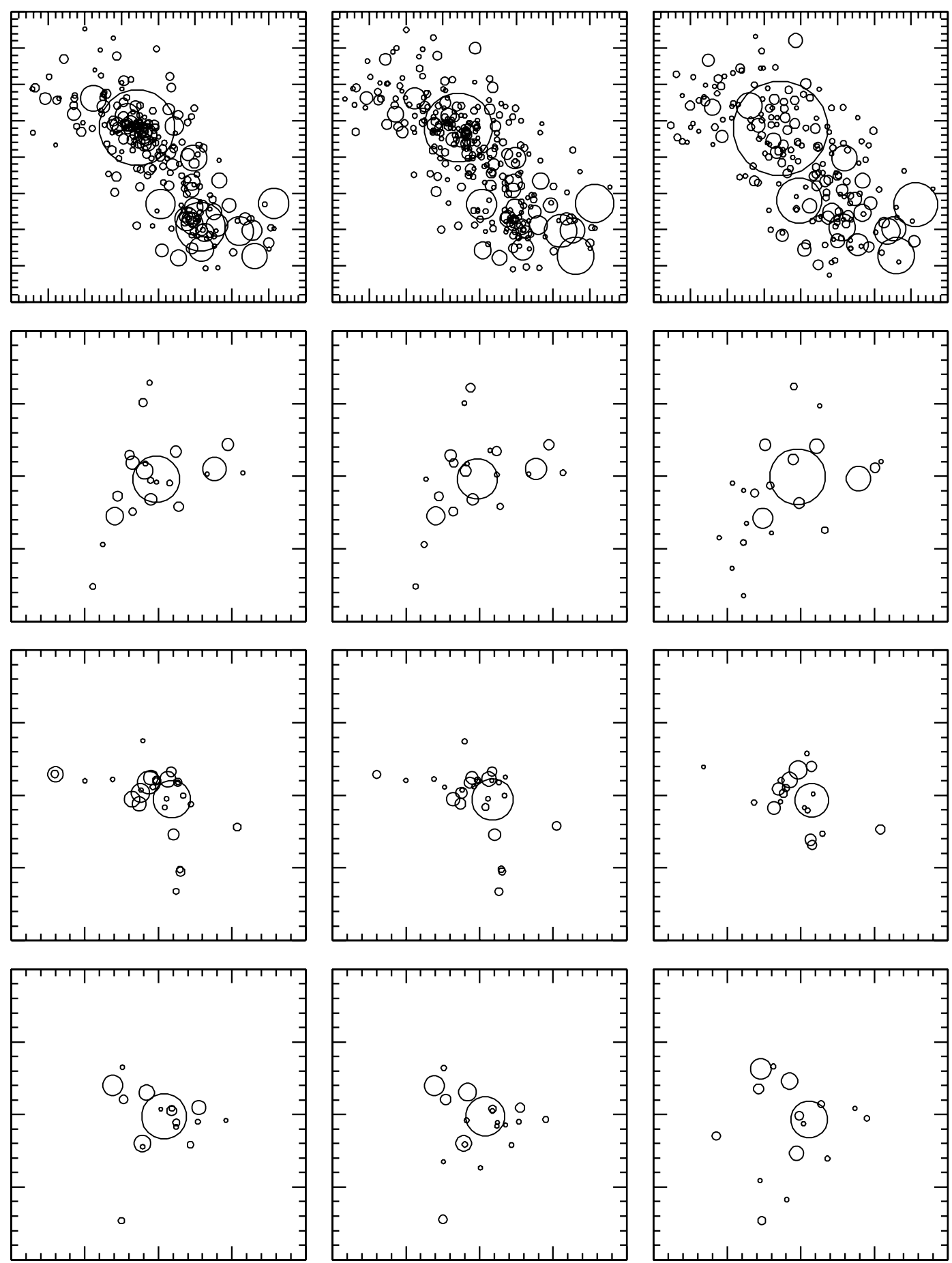

FIG. 4.-Galaxy and subhalo content of the four most massive halos. Left-hand panels show the SPH galaxies, middle panels show the dark matter subhalos in the SPH simulation, and right panels show the subhalos in the DM simulation. Each galaxy or subhalo is represented by a circle with an area proportional to its mass; the masses of the subhalos have been multiplied by 0.2 but are otherwise on the same scale as the galaxies. The top panels are $4 h^{-1}$ Mpc on a side, while the other panels are $2 h^{-1} \mathrm{Mpc}$ on a side.

Figure 4 compares the subhalo populations of the two simulations to the galaxy populations of the SPH simulation, this time for the four most massive halos. The bottom row shows the same halo whose formation history is illustrated in Figure 2. In the lefthand panels, each galaxy is represented by a circle whose area is proportional to its baryonic mass (stars plus cold gas). The smallest circles correspond to a mass of $7.1 \times 10^{9} M_{\odot}$, slightly above our mass resolution limit. The middle panels show subhalos of the SPH simulation represented in the same fashion, except that all subhalo masses have been lowered by a factor of 5 (and the same point-size scaling and minimum mass threshold have then been applied). Right-hand panels show the DM simulation's subhalo population, with the same factor of 5 mass scaling. The limiting subhalo mass corresponds to 40 dark matter particles. ${ }^{2}$

There is good agreement between the locations and scaled masses of the SPH galaxies and the DM subhalos in the SPH

\footnotetext{
${ }^{2}$ In this and all subsequent figures, we have multiplied the particle masses in the SPH simulation by $\Omega_{m} /\left(\Omega_{m}-\Omega_{b}\right)=1.134$, so that SPH and DM subhalos with the same number of particles are assigned the same mass.
} 

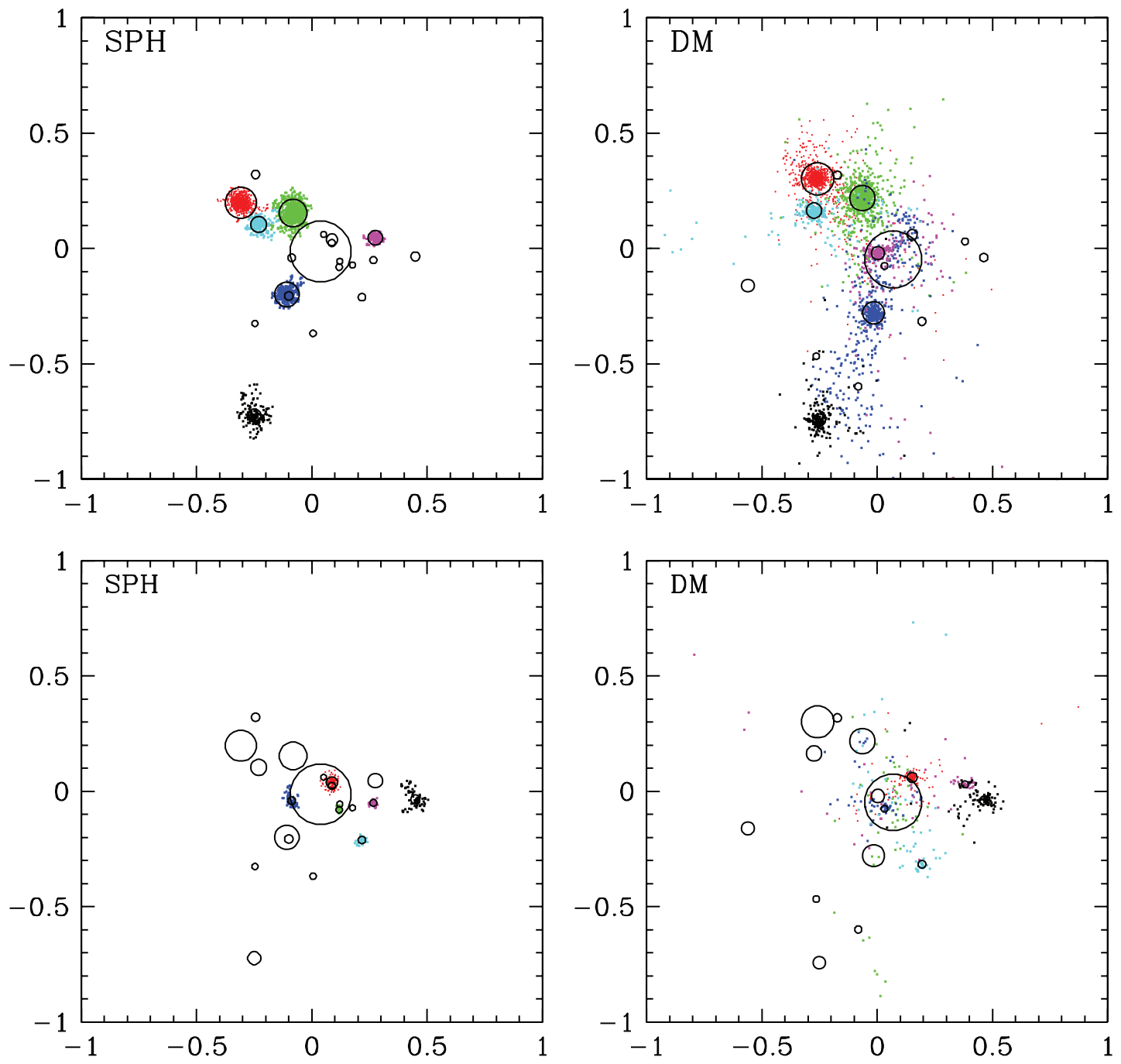

FIG. 5.-Correspondence of particles in subhalos of the fourth largest halo of the SPH simulation (left panels) and the DM simulation (right panels). Circles show subhalos of the two simulations as in the bottom panels of Fig. 4. Colored dots show particles associated with particular subhalos in the SPH simulation (left) and the locations of the corresponding particles in the DM simulation (right). In the top panels, the six most massive subhalos (after the most massive, central subhalo) are marked, while the bottom panels show lower mass subhalos that illustrate a range of behaviors.

simulation, and this agreement holds almost all the way to the resolution limit except in the most massive halo. More remarkably, there is good agreement between the locations and scaled masses of subhalos in the DM simulation and the galaxies (and subhalos) in the SPH simulation. There are some positional differences, and these become larger for lower mass subhalos, so at low masses it is difficult to tell whether there is still a one-to-one correspondence between subhalos in the two simulations.

Figures 5 and 6 show that the correspondence of subhalos remains good, although not perfect, even at fairly low masses. Figure 5 focuses on the fourth most massive halo, also shown in the bottom row of Figure 4. In the top left panel, small dots show the particles in six of the seven largest SPH subhalos, with a different color for each; we have omitted the largest subhalo to preserve visual clarity. Dots in the top right panel represent the corresponding particles in the DM simulation (i.e., those that had the same positions in the initial conditions), with the same color coding. In every case, there is a clear identification between an SPH subhalo and a DM subhalo, but particles in the DM simulation are more spread out. Since the subhalos are identified in the SPH simulation, it is virtually inevitable that the particle distributions will be more compact there, but the blue dotted halo, in particular, shows signs of substantial tidal stripping in the DM simulation. Of particular interest in this comparison is the magentadotted subhalo, which is at a significantly different location in the DM simulation but has much the same particle content, although it, too, shows signs of some tidal stripping.

The bottom panels of Figure 5 show similar results for six of the lower mass subhalos, ranging from 50 particles (black points) to 156 particles (red points). The black-, red-, and magentadotted subhalos have maintained their identity in the DM simulation, although the latter two have shifted positions noticeably. The cyan-dotted halo retains a core of the same particles at about the same location in the DM simulation, but many of its particles have been tidally stripped and are spread throughout the core of the halo. Finally, the blue-dotted and green-dotted halos appear to have been tidally disrupted, with their particle contents widely dispersed through the halo in the DM simulation.

Figure 6 shows quantitative results for the full halo population. For each SPH subhalo, we match a DM subhalo if it contains more than $\frac{1}{4}$ of the same particles. We suppress matches in which the DM subhalo is more than 4 times the mass of the SPH subhalo, since these cases arise when tidally stripped particles are attached to a different subhalo (typically the central one); the 

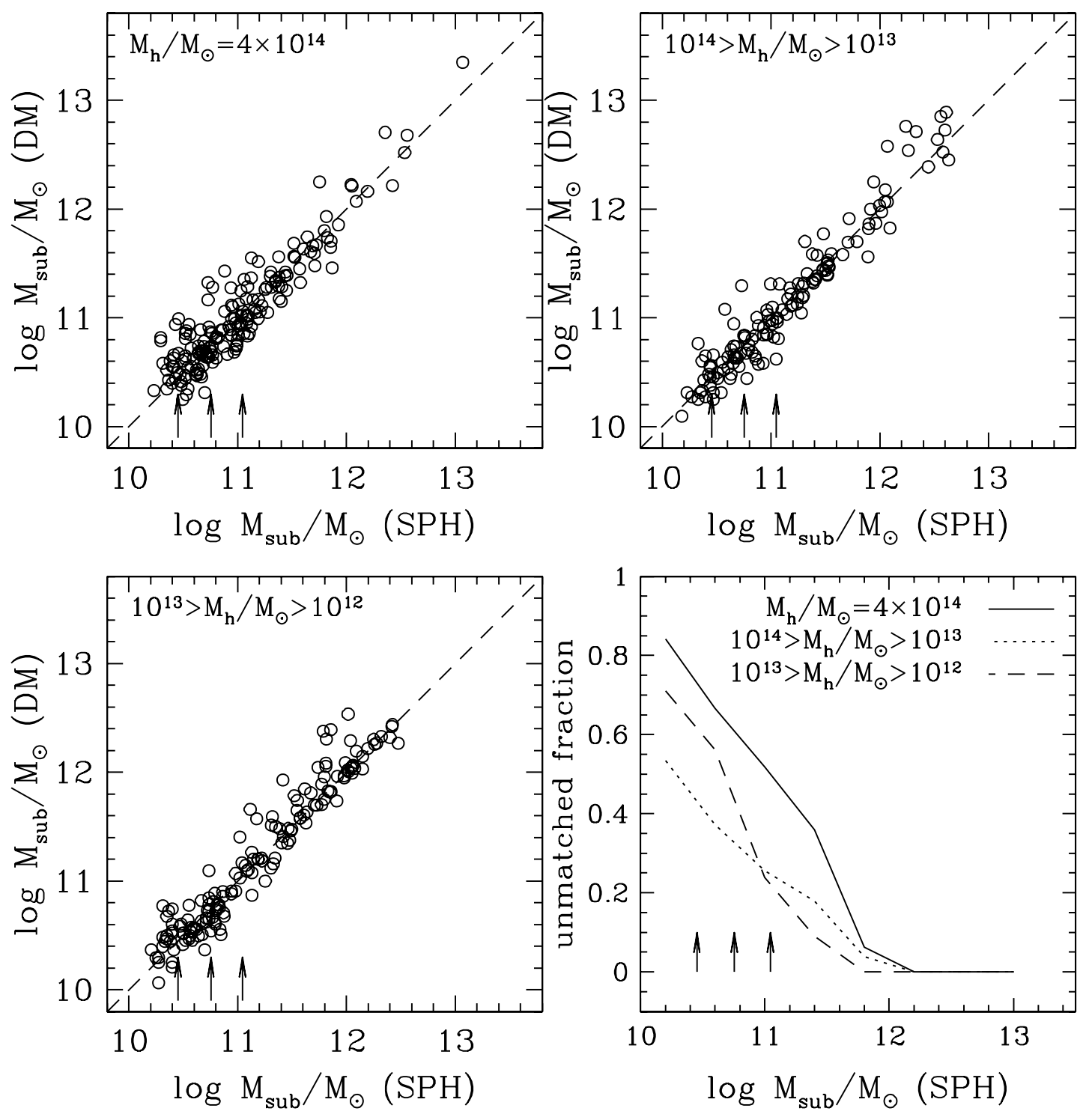

FIG. 6.-Masses of subhalos in the DM simulation vs. masses of the corresponding subhalos (see text) in the SPH simulation. Different panels show different host halo mass ranges as indicated. The bottom right panel shows the fraction of SPH subhalos that have no matching DM subhalo. Vertical arrows in each panel mark the masses corresponding to 32,64 , and 128 particles.

highest outliers in Figure 6 may be residual examples of such mismatches. The first three panels compare the masses of DM subhalos to masses of the matched SPH subhalos, in the $4 \times 10^{14} M_{\odot}$. halo (top left), the halos with $10^{13} M_{\odot}<M<10^{14} M_{\odot}$ (top right), and $10^{12} M_{\odot}<M<10^{13} M_{\odot}($ bottom left $)$. The agreement in subhalo masses is generally very good, with somewhat larger scatter for the least massive subhalos in the most massive halo. The bottom right panel shows the fraction of subhalos that are unmatched as a function of subhalo mass. For the most massive halo, this fraction rises to $50 \%$ for $M_{\text {sub }} \sim 10^{11} M_{\odot}$, corresponding to $\sim 128$ particles (rightmost vertical arrow). For the lower mass halos, the matched fraction is still $\sim 75 \%$ at this $M_{\text {sub }}$, and it does not fall to $50 \%$ until $\sim 64$ particles $\left(10^{13} M_{\odot}<M<10^{14} M_{\odot}\right)$ or $\sim 25$ particles $\left(10^{12} M_{\odot}<M<10^{13} M_{\odot}\right)$. It is not clear whether the "missing" subhalo matches are primarily a consequence of physical disruption at low masses or numerical artifacts at low particle number, but our results suggest that one should be cautious in interpreting subhalo mass functions in cluster simulations below $\sim 100$ particles or $\sim 10^{11} M_{\odot}$.
Returning to the top row of Figure 4, one can see in the densest regions of the largest halo a slight paucity of subhalos (relative to the galaxies) in the SPH simulation and a more substantial lack of subhalos in the DM simulation. Figure 7 compares the radial number density profiles of galaxies and subhalos around the central galaxy of the main component of this halo. Here we use a mass threshold of $7.1 \times 10^{9} M_{\odot}$ for the galaxies and a mass threshold larger by $\Omega_{\mathrm{dm}} / \Omega_{b}$ for the subhalos, where $\Omega_{\mathrm{dm}}=\Omega_{m}-$ $\Omega_{b}$. Since we normalize the profiles to the mean density of the corresponding galaxy or subhalo population in the entire simulation volume, the qualitative appearance of Figure 7 is not sensitive to the choice of mass thresholds. The radial profile of the SPH subhalos is only slightly depressed relative to the galaxies, but the DM subhalos are substantially depleted within $R \sim$ $0.2 h^{-1} \mathrm{Mpc}$. This result agrees with other recent studies of subhalo depletion in cluster mass halos (Diemand et al. 2004; Gao et al. 2004; Nagai \& Kravtsov 2005), although the impact of the baryons on the survival of subhalos appears somewhat stronger here than in Nagai \& Kravtsov (2005). Macciò et al. (2006) find a 


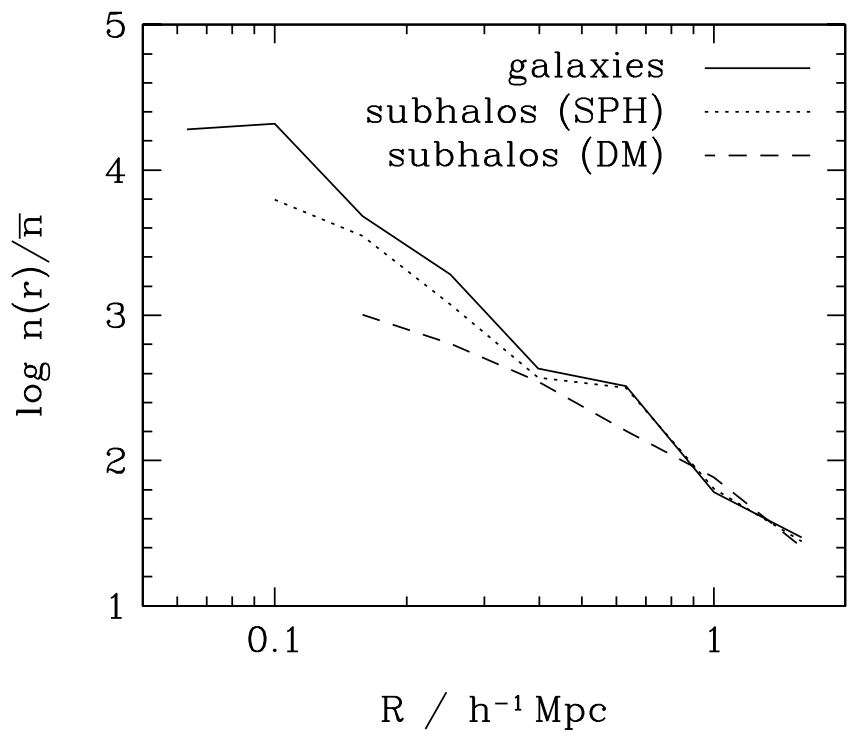

FIG. 7.-Radial number density profile of galaxies (solid line) and subhalos (dotted and dashed lines for the SPH and DM simulations, respectively) around the most massive galaxy of the most massive halo. Galaxies are selected above a mass threshold $M_{g, \min }=7.1 \times 10^{9} M_{\odot}$, and subhalos are selected above a mass threshold $M_{g, \min } \Omega_{\mathrm{dm}} / \Omega_{b}$. Densities are normalized to the mean density of galaxies or subhalos above the mass threshold in the full simulation volume. Curves stop when the only interior galaxy or subhalo is the central object.

similar effect for subhalos in a galaxy mass halo, again becoming prominent within $\sim 20 \%$ of the virial radius.

Can one use substructure in a high-resolution $N$-body simulation to identify the galaxy population that would be found in a full hydrodynamic simulation? Here we will focus on galaxy populations defined by thresholds in baryonic mass (stars plus cold, dense gas), which should be similar but not identical to populations defined by thresholds in luminosity. Two subtleties then arise in trying to answer the question. First, since halos retain an enormous amount of substructure if one goes to sufficiently small mass scales (e.g., Moore et al. 1999; Springel et al. 2001), it is virtually guaranteed that one can find "enough" substructures in each halo to correspond to the number of galaxies above a moderate or high baryonic mass threshold. However, the $\mathrm{N}$-body/ substructure approach has no predictive power unless one knows which (or at least how many) substructures to pick in each halo, so there must be some threshold in substructure mass for a given baryonic mass threshold.

The second subtlety arises because a halo that falls into a larger halo (and thus becomes a substructure) starts to lose mass via tidal stripping. Since this process does not remove mass from the halo's central galaxy (at least until it is close to total disruption), the mass of a substructure containing a galaxy in a group or cluster will generally be smaller than the mass of an isolated halo that contains a similar galaxy in the "field." It is therefore unlikely that a simple global threshold in substructure mass will work for identifying a galaxy population - if one picks the threshold based on the lowest mass halos that host such galaxies in the field, then there will be too few "galaxies" found in rich groups and clusters. One way to tackle this problem is to use circular velocity thresholds instead of mass thresholds, in the hope that the circular velocity remains a nearly monotonic function of the central galaxy's baryonic mass even if tidal stripping removes the outer parts of the halo in which the galaxy formed. This approach suffers from ambiguity in the choice of where to define the circular velocity, especially since tidal stripping alters the density profiles of substructures, making them systematically different from isolated halos (Stoehr et al. 2002). Furthermore, recent $N$-body studies indicate that the circular velocities of subhalos do in fact decline as they are tidally stripped (Nagai \& Kravtsov 2005), so using circular velocity instead of subhalo mass only partly compensates for stripping effects.

Here we have adopted a simple approach that seems to work surprisingly well. To identify a substructure population that corresponds to the galaxy population above mass threshold $M_{b, \min }$, we first apply a global mass threshold to the halo (not substructure) population. In the SPH simulation, we find the halo mass $M_{h, \text { min }}$ at which $50 \%$ of halos (in a sample of 20 centered on that mass) contain a galaxy above $M_{b, \min }$, and we eliminate halos with $M<M_{h, \min }$. We use the same threshold mass in the DM simulation, except that we multiply $M_{h, \text { min }}$ by $\Omega_{m} /\left(\Omega_{m}-\Omega_{b}\right)$ to account for the fact that baryons are not counted when computing the mass of the SPH halos. In the left-hand panel of Figure 8, crosses show the minimum halo mass (in the SPH simulation) as a function of the galaxy mass threshold. These lie close to the
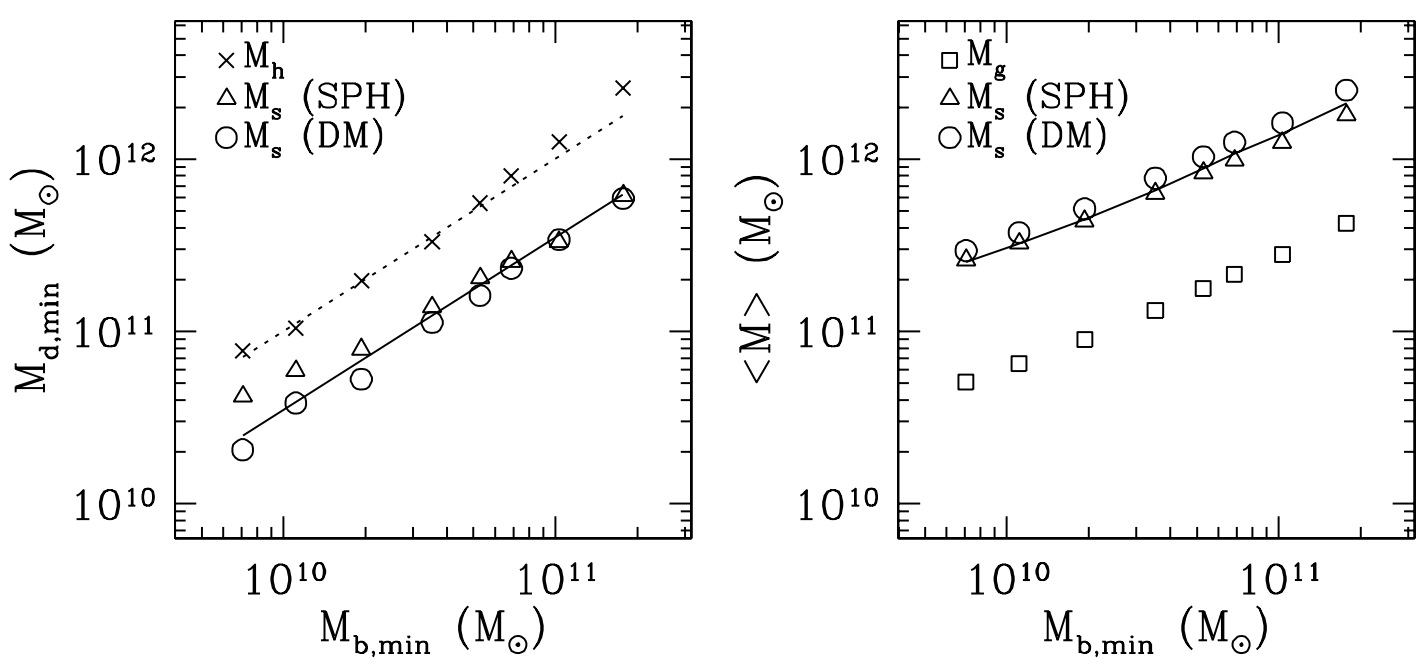

FIG. 8. - Left: Mass thresholds for identifying dark matter subhalos with SPH galaxies. Crosses show the halo mass $M$ at which $\langle N\rangle_{M}=0.5$ for galaxies above the threshold baryonic mass $M_{b, \min }$. When matching a subhalo population to a galaxy population, we only consider halos above this threshold mass. Triangles and circles show the subhalo mass threshold that is then required to match the space density of the galaxy population, in the SPH and DM simulations, respectively. For comparison, the dotted line shows $1.35 \times \Omega_{\mathrm{dm}} / \Omega_{b} \times M_{b, \min }$, and the solid line shows $3.5 M_{b, \min }$. Right: The average mass of galaxies (squares) above $M_{b, \text { min }}$ compared to the average mass of dark matter subhalos (triangles and circles for the SPH and DM simulations, respectively) above the mass threshold indicated in the left panel. The solid line shows the mean galaxy mass multiplied by 5 . 

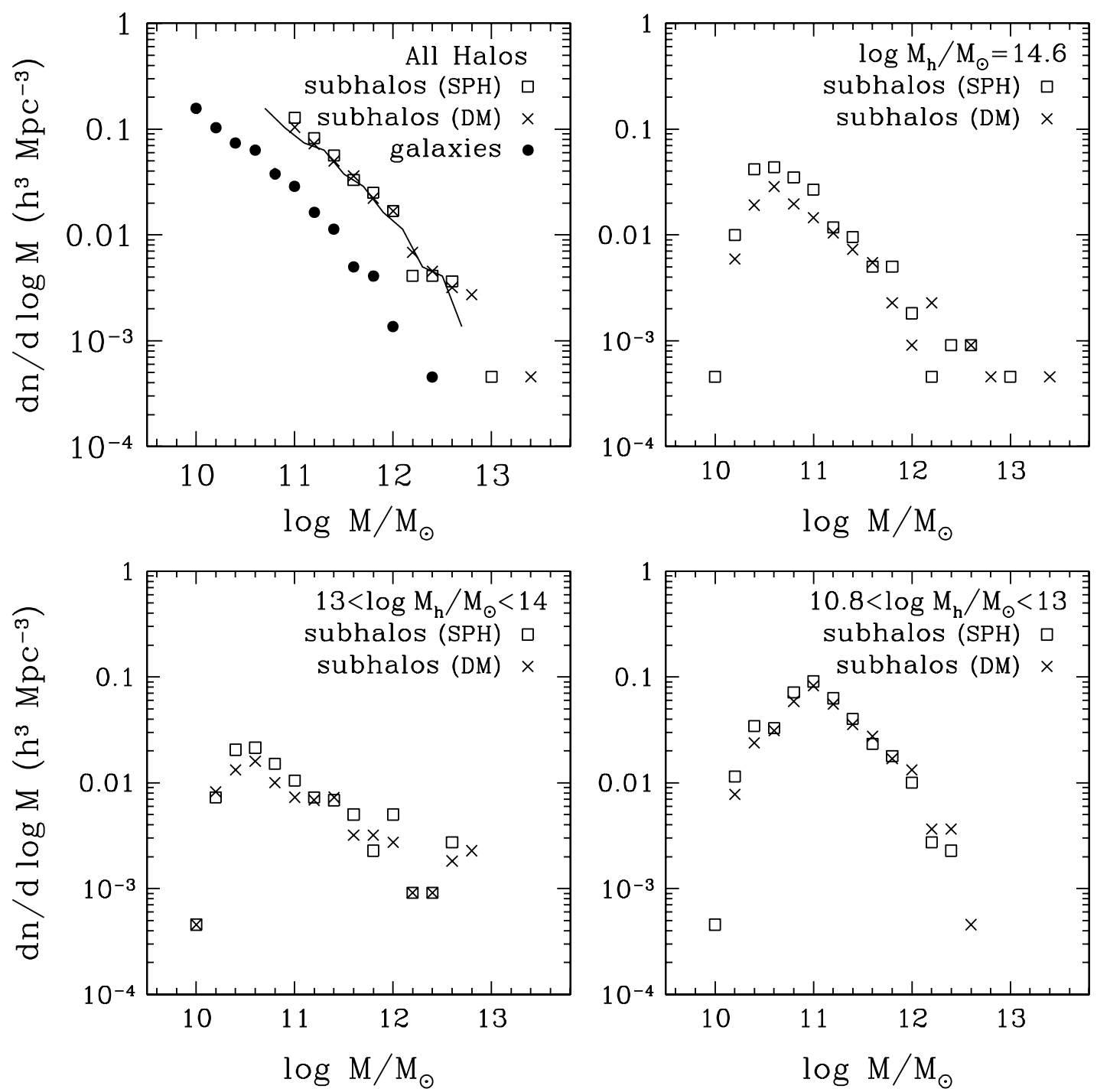

FIg. 9.-Differential mass functions, in number per $\left(h^{-1} \mathrm{Mpc}\right)^{3}$ per decade, of galaxies and subhalos. The top left panel shows results for the full simulation, with filled circles showing the baryonic mass function of SPH galaxies and squares and crosses showing the dark matter mass function of subhalos in the SPH and DM simulations, respectively. Here subhalos have been selected according to the two-stage thresholding procedure described in the text. The solid line shows the galaxy mass function shifted right by a factor of 5 . Note that bins are evenly spaced in $\log M$ but that some bins at high mass contain no objects. Remaining panels show the subhalo mass functions in bins of halo mass, as indicated. The lower limit of the $10.8<\log M_{h}<13$ bin corresponds to a mass of 64 dark matter particles. For these three panels we include all identified substructures, not just those selected by the thresholding procedure.

line $1.35\left(\Omega_{m}-\Omega_{b}\right) / \Omega_{b}$, indicating that these minimum mass halos have typically put about $75 \%$ of their available baryons into the central SPH galaxy.

After eliminating halos below the mass threshold, we now apply a global mass threshold $M_{s, \min }$ to the subhalo population, choosing its value so that the total number of subhalos in the simulation is equal to the total number of galaxies above the mass threshold. These subhalos are the "galaxy" population predicted by the subhalo method. The $M_{s, \min }$ threshold is always lower than $M_{h, \min }$ because of the tidal stripping effects discussed above. Note also that a halo that passes the $M_{h}$, min threshold may not, in the end, contain a galaxy, since the mass of its largest substructure may be lower than $M_{s, \min }$. These "unoccupied" halos (which are, in practice, fairly rare) should represent cases where the halo contains two or more galaxies below the $M_{b \text {, min }}$ threshold instead of one (or more) above it.

Triangles and circles in the left panel of Figure 8 show the threshold values required to match the galaxy and subhalo populations, for the SPH and DM simulations, respectively. The thresh- old for the DM simulation is approximately 3.5 times the galaxy baryonic mass, at all masses. The threshold for the SPH simulation is higher than this at low galaxy masses, suggesting that in this regime the baryonic clumps help to reduce tidal mass loss from their local subhalos. The right panel of Figure 8 shows the average masses of substructures above these thresholds. To a good approximation, the mean mass of subhalos above a threshold is simply 5 times the mean mass of galaxies above the corresponding threshold. We will show below that the halo occupation statistics and spatial clustering of the subhalo populations identified in this way are similar to those of the corresponding SPH galaxy populations. However, we first investigate the extent to which the presence of dissipative baryons in the SPH simulations alters the properties of the dark matter subhalos themselves.

\subsection{The Influence of Baryons on Halo Substructure}

Figure $9 a$ compares the differential baryonic mass function of SPH galaxies to the differential mass functions of subhalos in the SPH and DM simulations. The two subhalo mass functions are 


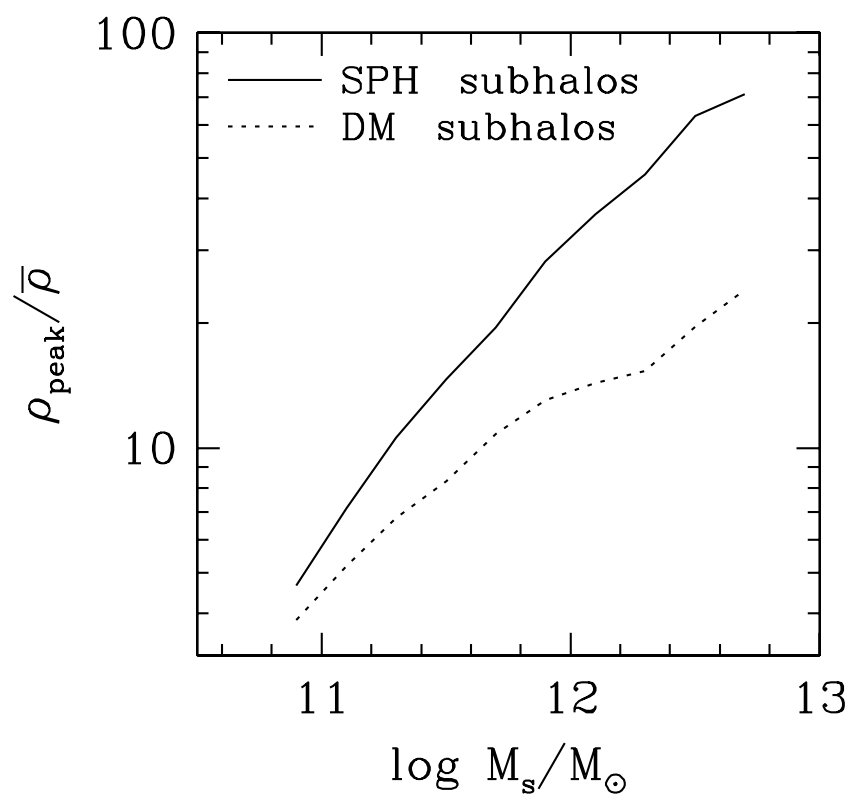

FIG. 10.-Average peak-to-mean density ratio of dark matter subhalos in the SPH simulation (solid line) and DM simulation (dotted line). Densities are computed for each particle in the subhalo using an SPH-like smoothing kernel containing 32 neighbors, and the quantity shown is the ratio of the maximum density in the subhalo to the mean density of the subhalo, averaged in bins of subhalo mass. The trend with subhalo mass is probably a mass resolution effect, but the difference between the SPH and DM simulations shows the concentrating effect of cooled baryonic components within subhalos.

similar, showing that the dissipative baryon component has only a small impact on this global measure of the subhalo population. The subhalo mass functions are similar in form to the galaxy mass function, shifted in mass scale by a factor of 5, with a somewhat larger shift at low masses.

The remaining panels show the subhalo mass function in the largest halo (Fig. $9 b$ ) and in halo mass ranges $\log M_{h} / M_{\odot}=13-$ 14 (Fig. 9c) and $10.8-13$ (Fig. 9d). Here we have not imposed any explicit threshold on the subhalo masses. The turnover of the subhalo mass functions at low masses is an artifact of the simulations' finite mass resolution, but since this resolution is the same in each case, we can use the differential comparison to investigate the influence of the baryon component on the survival of dark matter subhalos. These effects are generally mild, but they have the expected sign. In particular, the ability of dense baryon clumps to retain surrounding dark matter concentrations boosts the number of low-mass subhalos in the largest halos, by up to a factor of 2 .

Figure 10 examines the influence of baryons on the internal structure of subhalos. We define a simple measure of subhalo "concentration" by measuring a density for each particle using an SPH-like smoothing kernel containing 32 neighbors and taking the ratio of the highest density in the subhalo to the mean density of all particles in the subhalo. The strong trend of this concentration measure with subhalo mass is probably an effect of mass resolution - one can trace the density profile of more massive subhalos into smaller radii-but we can again make a differential comparison. In contrast to Figure 9, the influence of baryons on subhalo concentrations is strong, with differences of a factor of several by this measure. This result is in qualitative agreement with the expectation that dissipative baryons lead to adiabatic contraction of their surrounding dark matter concentrations (Blumenthal et al. 1986), although a quantitative investi-
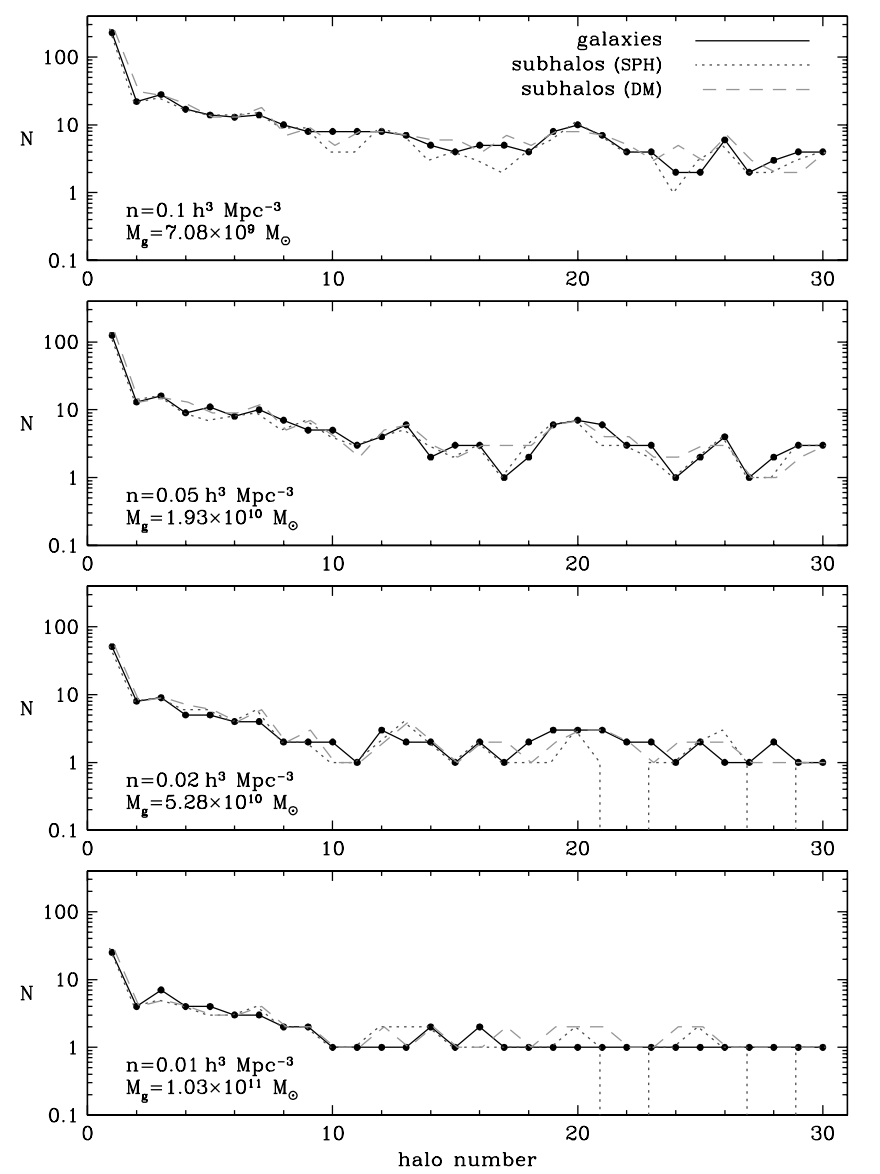

FIG. 11.-Halo occupations of the 30 most massive halos in the SPH and DM simulations. Points connected by the solid black line represent galaxies, while the dotted red and dashed green lines represent the matched subhalo populations in the SPH and DM simulations, respectively. From top to bottom, the four panels correspond to increasing galaxy baryonic mass thresholds and decreasing population space densities, as indicated in each panel. [See the electronic edition of the Journal for a color version of this figure.]

gation of these effects is better carried out with higher resolution simulations of individual halos (e.g., Gnedin et al. 2004).

\subsection{Halo Occupation Statistics}

On scales that are large compared to the virial diameters of the largest halos, the clustering of the galaxy population is determined by the number of galaxies in each halo, regardless of their internal distribution within halos. If the halo occupation statistics of a population of galaxies and a population of subhalos are identical, then they will yield the same large-scale results for all measures of clustering. Here we compare halo occupation statistics for mass-thresholded samples of SPH galaxies to those of matched subhalo samples defined by the two-stage thresholding procedure described in $\S 4.2$.

Points in Figure 11 show the number of galaxies in each of the 30 most massive halos of the SPH simulation. The four panels correspond to four different baryonic mass thresholds, and the mean space densities of galaxies above these thresholds are 0.1 , $0.05,0.02$, and $0.01 h^{3} \mathrm{Mpc}^{-3}$, respectively. Dotted lines show the number of subhalos in each of these halos above the mass thresholds indicated by the triangles in Figure $8 a$; by construction, the mean space density of these subhalos matches that of the corresponding galaxy population. Dashed lines show the number of subhalos in the same halos of the DM simulation, with mass 

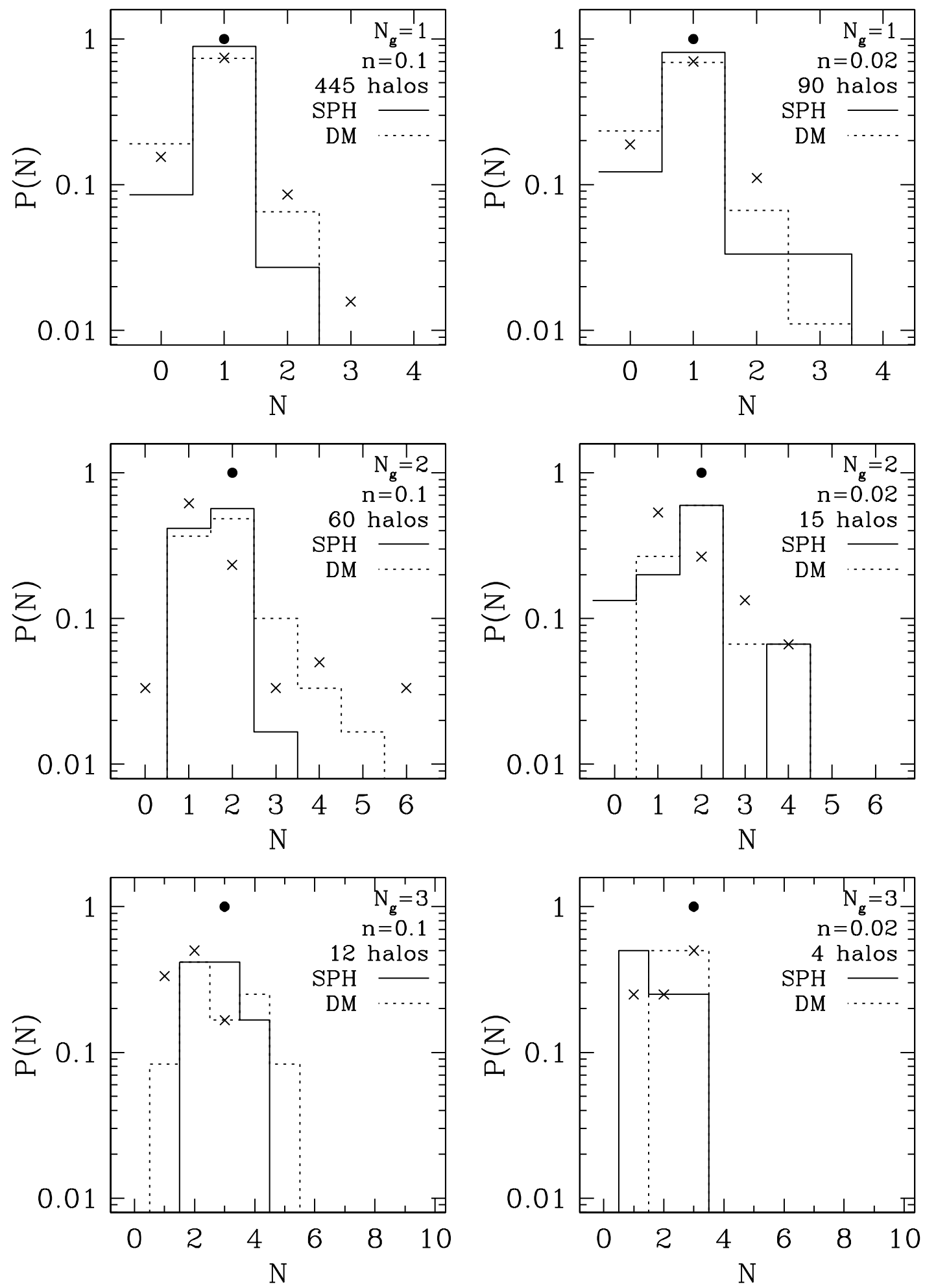

FIG. 12.-Distribution of subhalo occupations in halos with low galaxy occupation number. Top, middle, and bottom panels show results for halos that contain 1,2 , and $3 \mathrm{SPH}$ galaxies, respectively, in the $n=0.1 h^{3} \mathrm{Mpc}^{-3}$ sample (left) and the $n=0.02 h^{3} \mathrm{Mpc}^{-3}$ sample (right). Solid histograms show the distribution of the number of subhalos above the matching mass threshold in the SPH simulation, and dotted histograms show the same distribution for the DM simulation. Perfect agreement would correspond to $P(N)=1$ for $N=N_{g}$ and $P(N)=0$ otherwise, in which case the histograms would intersect the filled circles. Crosses show $P(N)$ for a "control" sample in which the galaxy occupation of each halo is replaced by the galaxy occupation of the next halo in a list rank-ordered by mass.

thresholds shown by the circles in Figure $8 a$. The agreement between the number of galaxies and the number of subhalos in the matched population is extraordinarily good for both the SPH and DM simulations, at all four space densities. This agreement holds for the most massive halo despite the visible paucity of subhalos in the densest regions of this halo (Fig. 4). The subhalo mass thresh- old, chosen to give agreement with the global number density of galaxies in the simulation, has the effect of replacing these missing subhalos in the halo core with slightly less massive subhalos in the outskirts.

Figure 12 examines the distribution of subhalo numbers in halos with $N_{g}=1,2$, or $3 \mathrm{SPH}$ galaxies (top to bottom), for 
samples with space densities of $0.1 h^{3} \mathrm{Mpc}^{-3}$ (left-hand panels) or $0.02 h^{3} \mathrm{Mpc}^{-3}$ (right-hand panels). In each panel, solid histograms show the distribution of subhalo numbers in the SPH simulation, and dotted histograms show the distribution in the DM simulation. If the subhalo and galaxy populations agreed perfectly, these histograms would be $P(N)=1$ for $N=N_{g}$ and $P(N)=0$ for all other $N$. The agreement is generally very good but not perfect. For example, of the 15 halos with $N_{g}=2$ for $n=$ $0.02 h^{3} \mathrm{Mpc}^{-3}$, nine have two subhalos in the DM simulation, four have one subhalo, one has three, and one has four. The agreement for the SPH subhalos is usually better than for the DM subhalos, but not dramatically so.

The detailed agreement seen in the individual halo plots of Figure 4 and in the number counts of Figure 11 suggests that the subhalo counts are not just reproducing the mean number of galaxies at a given halo mass but are, to some degree, tracking the variation in galaxy number from halo to halo at each mass. To investigate this issue in the low occupancy regime, we rankordered the halo list by mass, then replaced each halo's galaxy occupation $N_{g}$ with the occupation $N_{g}^{\prime}$ of the next halo on the list. Crosses in Figure 12 show $P\left(N_{g}^{\prime}\right)$ - in essence, they show the effect of randomly replacing each halo's galaxy population with that of another halo of nearly identical mass. The subhalo method is doing "better than random" if the histogram lies above the cross in the $N=N_{g}$ bin and below the crosses in the other bins. For $N_{g}=2$, this is clearly the case; one can predict a halo's galaxy number more accurately using its SPH or DM subhalos than by using the galaxy number of another halo of similar mass. For $N_{g}=1$ and $N_{g}=3$, on the other hand, subhalos do at most slightly better than random assignment. Note, however, that the absolute level of agreement for $N_{g}=1$ is high, and that fewer than $10 \%$ of halos that have two subhalos above threshold contain only a single galaxy.

Figure 13 compares the mean occupation functions $\langle N\rangle_{M}$ of the two galaxy samples to those of the corresponding subhalo samples. The locations of the lower cutoffs match by construction, since we eliminate halos below the mass at which $\langle N\rangle_{M}=$ 0.5 before choosing the subhalo population. In addition, matching the global space density of the subhalo population to that of the galaxy population forces agreement in the values of $\int_{0}^{\infty} d n / d M\langle N\rangle_{M} d M$, where $d n / d M$ is the halo mass function. However, it is clear that the agreement between the galaxy and subhalo occupation functions is far better than these constraints alone would require. The excellent match in halo occupations seen here and in Figure 11 implies that the large-scale clustering of a mass-thresholded galaxy population and a properly matched subhalo population should be very similar in all respects.

On small scales, the two-point correlation function is dominated by pairs of galaxies that reside in the same halo. In this onehalo regime, the important quantity for determining the two-point correlation function is $\langle N(N-1)\rangle_{M}$, the mean number of pairs per halo (Seljak 2000). Similarly, the three-point correlation function on small scales depends on the mean number of triples per halo $\langle N(N-1)(N-2)\rangle_{M}$ (Scoccimarro et al. 2001). Figures $14 a$ and $14 b$ plot $\langle N(N-1)\rangle_{M}^{1 / 2}$ and $\langle N(N-1)(N-2)\rangle_{M}^{1 / 3}$, respectively. Taking square and cube roots allows direct comparison to the mean occupations plotted in Figure 13: if $P\left(N \mid N_{\text {avg }}\right)$ is Poisson distributed, then $\langle N(N-1)(N-2)\rangle_{M}^{1 / 3}=\langle N(N-1)\rangle_{M}^{1 / 2}=\langle N\rangle_{M}$. Figure 14 shows good agreement between the pair and triple counts of galaxy and subhalo populations in all cases. In particular, the subhalo counts reproduce a key feature of the galaxy counts, namely, a distribution that is substantially narrower than a Poisson distribution at low occupation numbers. Berlind et al. (2003) and Kravtsov et al. (2004) discuss the physics behind these sub-

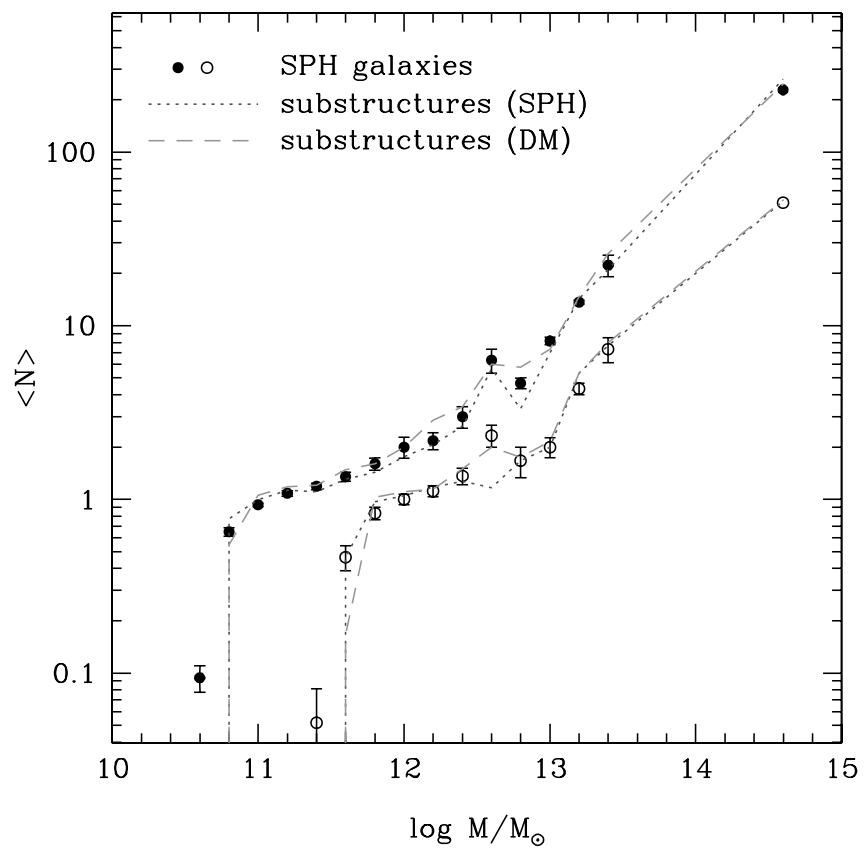

FIG. 13.--Mean number of galaxies ( points with error bars) and substructures (SPH simulation, dotted line; DM simulation, dashed line) in halos of mass $M$. Filled circles and upper lines represent the $n=0.1 h^{3} \mathrm{Mpc}^{-3}$ sample, while open circles and lower lines represent the $n=0.02 h^{3} \mathrm{Mpc}^{-3}$ sample. Error bars show the error on the mean, computed from the dispersion of $N$ among all halos in the bin divided by the square root of the number of halos. The highest mass bin contains only a single halo, so no error bar is computed. [See the electronic edition of the Journal for a color version of this figure.]

Poisson count distributions: over the range in which $\langle N\rangle$ rises from one to a few, more massive halos tend to host more massive central galaxies or subhalos instead of hosting multiple objects above the mass threshold (see also Benson et al. 2000; Zheng et al. 2005).

In the one-halo regime, galaxy clustering depends on the internal distribution of galaxies within halos, in addition to $P(N \mid M)$. Therefore, agreement in $\langle N(N-1)\rangle_{M}$ and $\langle N(N-1)(N-2)\rangle_{M}$ does not guarantee agreement in two- and three-point correlations on small scales. We now turn to a direct investigation of small-scale clustering as measured by the two-point correlation function and moments of counts-in-cells.

\section{5. $\xi(r)$ and $S_{n}$}

Because of the small simulation volume, the calculated galaxy clustering statistics are not good estimates of the global predictions for this cosmological model. However, since the DM and SPH simulations started from identical initial conditions, we can carry out differential comparisons between the clustering of SPH galaxies, SPH subhalos, and DM subhalos, and we expect the differences to be indicative of those that would arise in larger volumes.

Figure 15 shows the two-point correlation functions of SPH galaxies and subhalos, for samples with a space density of $0.1 h^{3} \mathrm{Mpc}^{-3}$ (left panel) and $0.02 h^{3} \mathrm{Mpc}^{-3}$ (right panel). The strong curvature in the range $r \sim 0.1-2 h^{-1} \mathrm{Mpc}$, especially evident for the $0.1 h^{3} \mathrm{Mpc}^{-3}$ sample, is produced by the single large halo; if we eliminate all members of this halo before computing $\xi(r)$, then the correlation functions have an approximately power-law form. At $r \gtrsim 0.5 h^{-1} \mathrm{Mpc}$, results for the galaxies, SPH subhalos, and DM subhalos converge, as expected based on the similarity of halo occupations shown in $\S 4$ 4. At smaller scales, the two-point function of subhalos is depressed relative to 

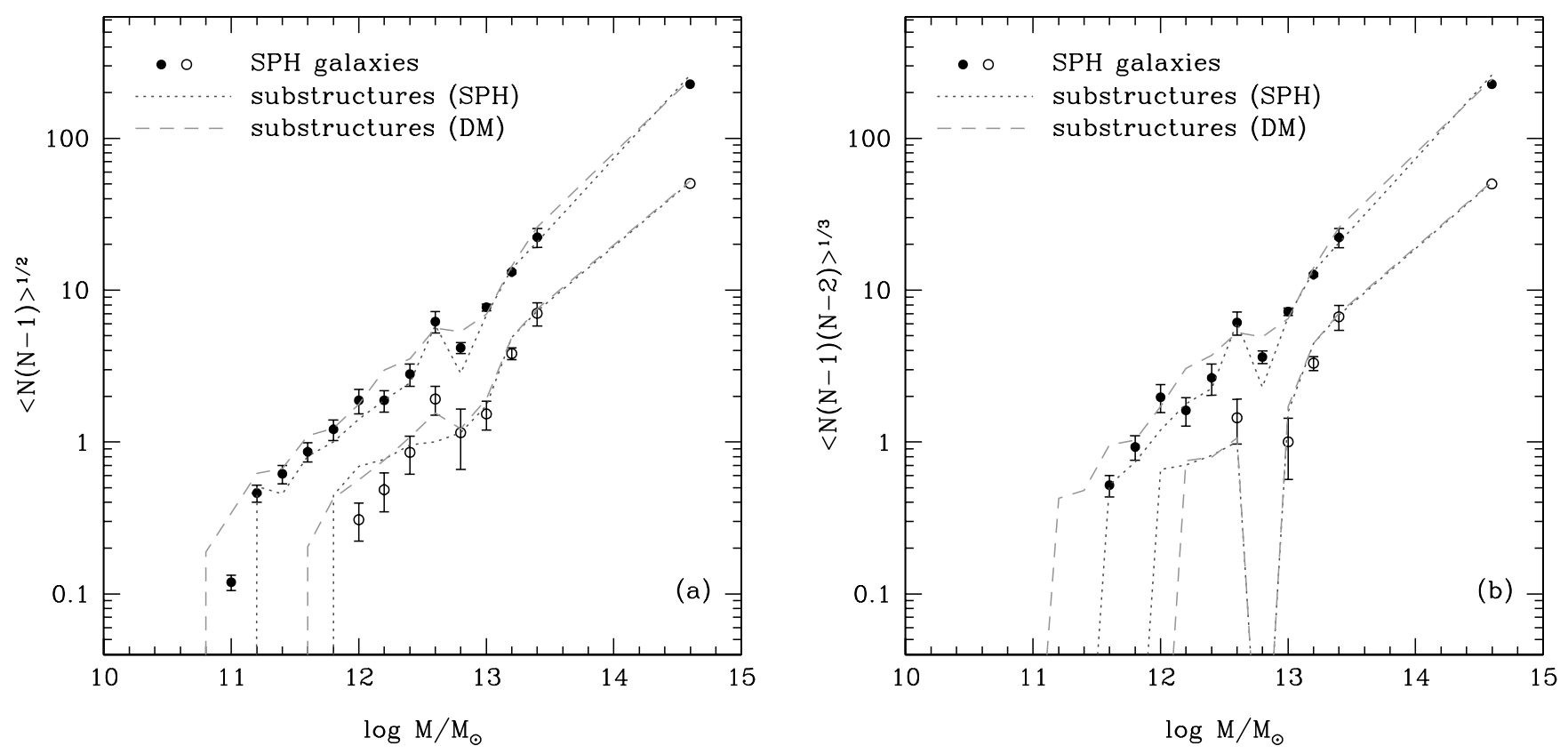

FIG. 14.-Left: The square root of the mean number of pairs per halo, $\langle N(N-1)\rangle_{M}^{1 / 2}$, in the same format as Fig. 13. Right: The cube root of the mean number of triples per halo, $\langle N(N-1)(N-2)\rangle_{M}^{1 / 3}$. [See the electronic edition of the Journal for a color version of this figure.]

that of galaxies, more strongly for the subhalos of the DM simulation, and more strongly for the sample with lower mass threshold $\left(n=0.1 h^{3} \mathrm{Mpc}^{-3}\right)$. This departure is primarily caused by the depletion of substructures in the densest regions of the largest halo, as seen in Figure 4. If we omit this halo, then the correlation functions of galaxies and subhalos track each other down to $0.05 h^{-1} \mathrm{Mpc}$ for $n=0.1 h^{3} \mathrm{Mpc}^{-3}$ and to $0.2 h^{-1} \mathrm{Mpc}$ for $n=0.02 h^{3} \mathrm{Mpc}^{-3}$, below which the number of pairs is too small for reliable comparison. Colín et al. (1999) and Kravtsov et al. (2004) find subhalo correlation functions that retain an approximately power-law form down to $r \sim 0.05 h^{-1} \mathrm{Mpc}$. Since

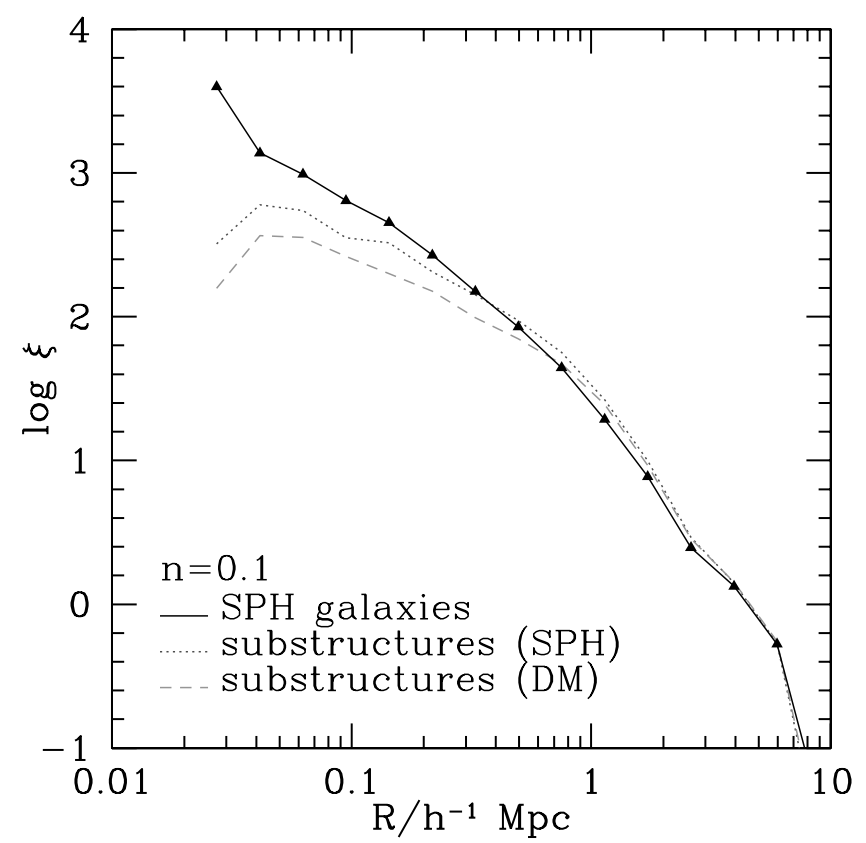

depletion of substructure is important mainly in the largest halos, the difference between their results and those shown in Figure 15 can be explained in large part by the anomalously large contribution that the largest halo in our simulation volume makes to $\xi(r)$. Furthermore, the depletion of subhalos in our $n=0.1 h^{3} \mathrm{Mpc}^{-3}$ sample could be artificially enhanced by numerical effects, since the subhalo mass thresholds for this sample correspond to only 53 and 23 dark matter particles in the SPH and DM simulations, respectively.

As a simple measure of higher order correlations, Figure 16 shows the hierarchical moment ratios $S_{3}, S_{4}$, and $S_{5}$ in spherical

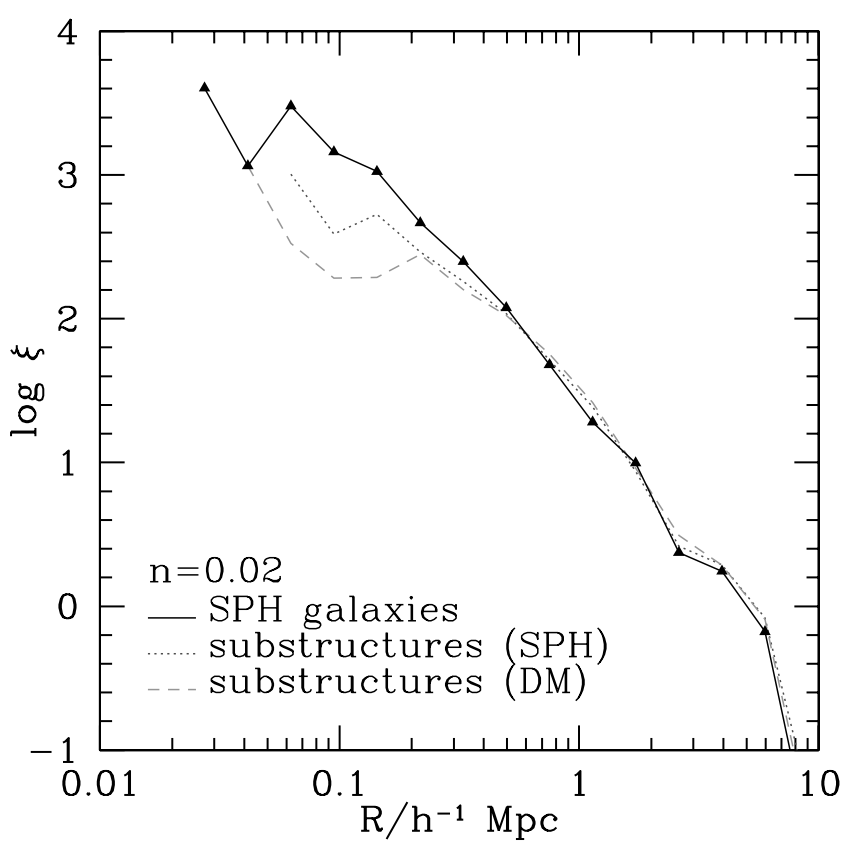

FIG. 15.-Two-point correlation function of SPH galaxies (solid line), SPH subhalos (dotted line), and DM subhalos (dashed line). The left-hand panel shows samples with a space density of $0.1 h^{3} \mathrm{Mpc}^{-3}$, and the right-hand panel shows samples with higher mass thresholds and a space density of $0.02 h^{3} \mathrm{Mpc}^{-3}$. [See the electronic edition of the Journal for a color version of this figure.] 

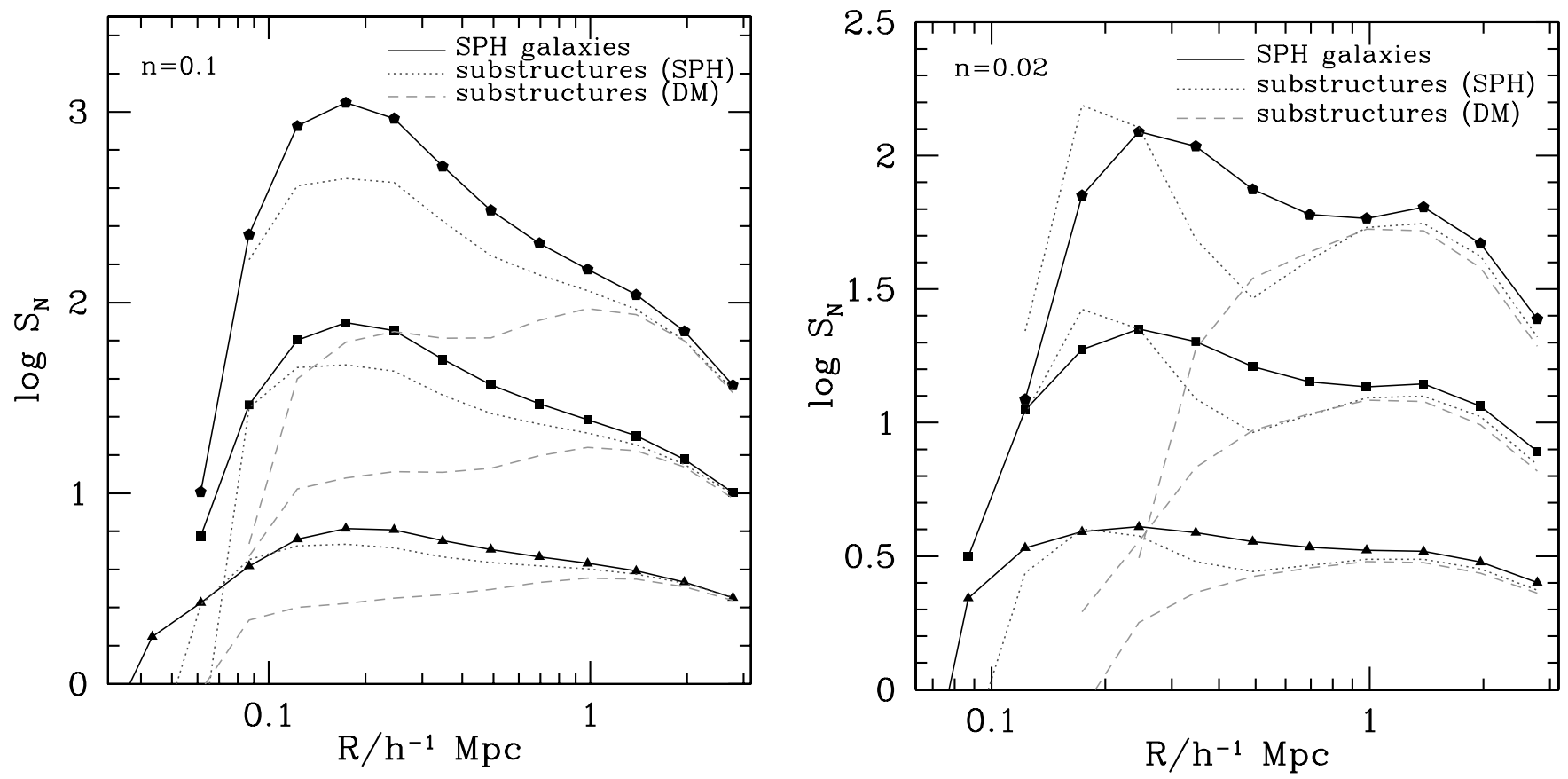

Fig. 16. - Hierarchical moment ratios $S_{3}, S_{4}$, and $S_{5}$ (bottom to top) for SPH galaxies (solid lines), SPH subhalos (dotted lines), and DM subhalos (dashed lines). The lefthand panel shows samples with a space density of $0.1 \mathrm{~h}^{3} \mathrm{Mpc}^{-3}$, and the right-hand panel shows samples with higher mass thresholds and a space density of $0.02 h^{3} \mathrm{Mpc}^{-3}$.

cells, estimated from shot-noise corrected moments of the galaxy and subhalo count distributions in $2048^{3}$ cells. Here $S_{n} \equiv$ $\left\langle\delta_{c}^{n}\right\rangle /\left\langle\delta^{2}\right\rangle^{n-1}$, where $\left\langle\delta_{c}^{n}\right\rangle$ is the $n$th connected moment of the density field and $\left\langle\delta^{2}\right\rangle$ is the variance, both corrected for shotnoise (see, e.g., Szapudi \& Szalay 1993). As with the two-point correlation function, the hierarchical moment ratios of galaxies and subhalos converge at scales $R \gtrsim 1 h^{-1} \mathrm{Mpc}$, but they differ substantially at smaller scales, with SPH substructures slightly depressed and DM substructures more substantially depressed. Statistics for the $n=0.02 h^{3} \mathrm{Mpc}^{-3}$ sample are poor, but for the $n=0.1 h^{3} \mathrm{Mpc}^{-3}$ sample, the depletion of substructure in the densest regions clearly has an increasing impact for higher moments of the density field, as one might expect. The convergence scale is larger for moment ratios (and for the variance of countsin-cells) than for $\xi(r)$ because the moments in cells of radius $R$ are affected by structure on all scales smaller than $R$.

\section{DISCUSSION}

Our comparison of matched SPH and $N$-body simulations illuminates several aspects of the baryon-dark matter "conversation." For the most part, this conversation is one sided: the dark matter talks, and the baryons listen. If one traces the history of a halo back in time (Fig. 2), then the population of the progenitor halos, where gas condenses into galaxies, is nearly identical in simulations with and without gas. At $z=0$, the positions and masses of the larger subhalos in each halo are similar between the two simulations. Smaller subhalos have different positions, presumably because small differences in the halo potential can modify their orbits, but in many cases even these lower mass subhalos can be matched based on particle memberships. Some SPH subhalos are tidally stripped or disrupted in the DM simulation. However, the mass function of subhalos is similar in the two cases, with baryonic effects producing only a modest enhancement, primarily for low-mass subhalos in high-mass halos. The dissipative baryon component does increase the internal density of subhalos, and while this has little effect on their masses in typical environments, it does enhance the survival of subhalos in the densest regions of massive halos.

These results are not especially surprising, but they are certainly at the simple end of what might have been expected. Our most remarkable result is the success of a simple mass thresholding scheme in identifying subhalo populations that have nearly identical halo occupation statistics to SPH galaxy populations with the same mean space density. To some degree, the number of subhalos traces the variation of galaxy number in halos of fixed mass; in particular, halos with two significant subhalos are more likely to host two significant galaxies. For each galaxy mass threshold, the average mass of host subhalos is approximately 5 times the average baryonic mass of the galaxies themselves (of course, the value of this ratio is likely to depend on the adopted cosmological parameters).

Our results have encouraging implications for efforts to model galaxy clustering with the subhalo populations of high resolution, dissipationless simulations (e.g., Colín et al. 1999; Kravtsov \& Klypin 1999; Conroy et al. 2006) and to develop semiempirical models of galaxy bias by monotonically matching galaxy luminosity functions to subhalo mass functions (Vale \& Ostriker 2004, 2006). The agreement in halo occupation statistics implies that SPH galaxies and dark matter subhalos should have similar largescale clustering statistics, and we indeed find good agreement in the two-point correlation function and the hierarchical moments $S_{3}, S_{4}$, and $S_{5}$ on scales larger than $\sim 1 h^{-1}$ Mpc. However, we find significant depletion of dark matter substructure in the densest regions of our one cluster mass halo, in agreement with results from other groups (Diemand et al. 2004; Gao et al. 2004; Nagai \& Kravtsov 2005). This depletion significantly affects clustering on scales $\leqslant 1 h^{-1} \mathrm{Mpc}$, with galaxy clustering stronger than SPH subhalo clustering, which in turn is stronger than DM subhalo clustering. The impact on these global statistics might be exaggerated in our simulations by the dominance of the largest halo in our small volume. Conversely, since the depletion is most likely a function of the ratio of subhalo mass to virial mass (see, 
e.g., Macciò et al. 2006), the impact on the clustering of faint galaxies could be more important, since they reside in subhalos smaller than those resolved here.

Nagai \& Kravtsov (2005) note that subhalo and galaxy density profiles are more similar if one selects subhalos based on the mass they have when they are accreted onto the main halo, rather than the final mass, which is preferentially reduced by tidal stripping in the inner regions. In the densest regions of our most massive $\mathrm{N}$-body halo, the dark matter distribution is quite smooth, so simply rescaling the masses of identifiable subhalos would not restore the galaxies missing from these regions. However, a higher resolution simulation might retain more low-mass substructures, allowing a rescaling based on accreted mass to have larger effect. Conroy et al. (2006) show that selecting subhalos based on accreted mass yields good agreement with observed galaxy clustering over a wide range of redshifts and luminosities.
Overall, we find that the present day distribution of dark matter gives one a good idea of where to place galaxies, and the relation between subhalo mass and galaxy baryon mass is roughly monotonic even if one uses the final subhalo mass. Simple recipes for matching galaxy and subhalo populations fail in the densest environments, but the large-scale clustering of galaxies is determined mainly by the gravitational dynamics of dark matter.

We thank Andrey Kravtsov for informative discussions on the topic of subhalo clustering. This research was supported by NASA grant NAGS-13308, NSF grant AST 04-07125, and the French CNRS. D. H. W. thanks the Institut d'Astrophysique de Paris for generous hospitality during the key phases of this work.
Aubert, D., Pichon, C., \& Colombi, S. 2004, MNRAS, 352, 376

Benson, A. J., Cole, S., Frenk, C. S., Baugh, C. M., \& Lacey, C. G. 2000 , MNRAS, 311, 793

Berlind, A. A., \& Weinberg, D. H. 2002, ApJ, 575, 587

Berlind, A. A., et al. 2003, ApJ, 593, 1

Blumenthal, G. R., Faber, S. M., Flores, R., \& Primack, J. R. 1986, ApJ, 301, 27

Cen, R., \& Ostriker, J. P. 2000, ApJ, 538, 83

Cole, S., Lacey, C. G., Baugh, C. M., \& Frenk, C. S. 2000, MNRAS, 319, 168 Colín, P., Klypin, A. A., Kravtsov, A. V., \& Khokhlov, A. M. 1999, ApJ, 523, 32

Conroy, C., Wechsler, R. H., \& Kravtsov, A. V. 2006, ApJ, 647, 201

Davé, R., Dubinski, J., \& Hernquist, L. 1997a, NewA, 2, 277

Diemand, J., Moore, B., \& Stadel, J. 2004, MNRAS, 352, 535

Eisenstein, D. J., \& Hut, P. 1998, ApJ, 498, 137

Gao, L., White, S. D. M., Jenkins, A., Stoehr, F., \& Springel, V. 2004, MNRAS, 355,819

Gelb, J. M., \& Bertschinger, E. 1994, ApJ, 436, 467

Ghigna, S., Moore, B., Governato, F., Lake, G., Quinn, T., \& Stadel, J. 1998, MNRAS, 300, 146

Gnedin, O. Y., Kravtsov, A. V., Klypin, A. A., \& Nagai, D. 2004, ApJ, 616, 16

Haardt, F., \& Madau, P. 1996, ApJ, 461, 20

Hernquist, L., \& Katz, N. 1989, ApJS, 70, 419

Katz, N., Weinberg, D. H., \& Hernquist, L. 1996, ApJS, 105, 19 (KWH)

Keres, D., Katz, N., Davé, R., \& Weinberg, D. H. 2005, MNRAS, 363, 2

Klypin, A., Gottlöber, S., Kravtsov, A. V., \& Khokhlov, A. M. 1999, ApJ, 516, 530

Kravtsov, A. V., Berlind, A. A., Wechsler, R. H., Klypin, A. A., Gottloeber, S., Allgood, B., \& Primack, J. R. 2004, ApJ, 609, 35

\section{REFERENCES}

Kravtsov, A. V., \& Klypin, A. 1999, ApJ, 520, 437

Macciò, A. V., Moore, B., Stadel, J., \& Diemand, J. 2006, MNRAS, 366, 1529

Moore, B., Quinn, T., Governato, F., Stadel, J., \& Lake, G. 1999, MNRAS, 310,1147

Nagai, D., \& Kravtsov, A. V. 2005, ApJ, 618, 557

Pearce, F. R., Jenkins, A., Frenk, C. S., White, S. D. M., Thomas, P. A., Couchman, H. M. P., Peacock, J. A., \& Efstathiou, G. 2001, MNRAS, 326, 649

Sánchez, A. G., Baugh, C. M., Percival, W. J., Peacock, J. A., Padilla, N. D.,

Cole, S., Frenk, C. S., \& Norberg, P. 2006, MNRAS, 366, 189

Scoccimarro, R., Sheth, R. K., Hui, L., \& Jain, B. 2001, ApJ, 546, 20

Seljak, U. 2000, MNRAS, 318, 203

Seljak, U., Makarov, A., McDonald, P., Anderson, S., et al. 2005, Phys. Rev. D, 71,3515

Spergel, D. N., et al. 2003, ApJS, 148, 175

Springel, V., White, S. D. M., Tormen, G., \& Kauffmann, G. 2001, MNRAS, 328,726

Springel, V., et al. 2005, Nature, 435, 629

Stoehr, F., White, S. D. M., Tormen, G., \& Springel, V. 2002, MNRAS, 335, L84

Szapudi, I., \& Szalay, A. S. 1993, ApJ, 408, 43

Tegmark, M., et al. 2004, Phys. Rev. D, 69, 103501

Vale, A., \& Ostriker, J. P. 2004, MNRAS, 353, 189

. 2006, MNRAS, 371, 1173

Weinberg, D. H., Davé, R., Katz, N., \& Hernquist, L. 2004, ApJ, 601, 1

White, S. D. M., \& Rees, M. J. 1978, MNRAS, 183, 341

Yoshikawa, K., Taruya, A., Jing, Y. P., \& Suto, Y. 2001, ApJ, 558, 520

Zheng, Z., Berlind, A. A., Weinberg, D. H., Benson, A. J., Baugh, C. M., Cole, S., Davé, R., Frenk, C. S., Katz, N., \& Lacey, C. G. 2005, ApJ, 633, 791 